\title{
Ages and luminosities of young SMC/LMC star clusters and the recent star formation history of the Clouds $\star$
}

\author{
K. Glatt ${ }^{1,2}$, E. K. Grebel ${ }^{1,2}$, and A. Koch ${ }^{3}$
}

\author{
1 Department of Physics and Astronomy, Klingelbergstrasse 82, 4056 Basel, Switzerland \\ e-mail: kglatt@ari.uni-heidelberg.de \\ 2 Astronomisches Rechen-Institut, Zentrum für Astronomie der Universität Heidelberg, Mönchhofstr. 12-14, 69120 Heidelberg, \\ Germany \\ 3 Department of Physics and Astronomy, University of Leicester, University Road, Leicester, LE1 7RH, UK
}

Received 3 February 2010 / Accepted 26 March 2010

\section{ABSTRACT}

\begin{abstract}
Aims. In this paper we discuss the age and spatial distribution of young (age $<1$ Gyr) Small Magellanic Cloud (SMC) and Large Magellanic Cloud (LMC) clusters using data from the Magellanic Cloud Photometric Surveys. Luminosities are calculated for all age-dated clusters.

Methods. The ages of 324 and 1193 populous star clusters in the SMC and the LMC were determined fitting Padova and Geneva isochrone models to their resolved color-magnitude diagrams. The clusters cover an age range between $10 \mathrm{Myr}$ and $1 \mathrm{Gyr}$ in each galaxy. For the SMC, a constant distance modulus of $(m-M)_{0}=18.90$ and a metallicity of $Z=0.004$ were adopted. For the LMC, we used a constant distance modulus of $(m-M)_{0}=18.50$ and a metallicity of $Z=0.008$. For both galaxies, we used a variable color excess to derive the cluster ages.

Results. We find two periods of enhanced cluster formation in both galaxies at $160 \mathrm{Myr}$ and $630 \mathrm{Myr}$ (SMC) and at $125 \mathrm{Myr}$ and $800 \mathrm{Myr}$ (LMC). We present the spatially resolved recent star formation history of both Clouds based on young star clusters. The first peak may have been triggered by a close encounter between the SMC and the LMC. In both galaxies, the youngest clusters reside in the supergiant shells, giant shells, the intershell regions, and toward regions with a high $\mathrm{H} \alpha$ content, suggesting that their formation is related to expansion and shell-shell interaction. Most of the clusters are older than the dynamical age of the supergiant shells. No evidence of cluster dissolution was found. Computed $V$ band luminosities show a trend toward fainter magnitudes with increasing age, as well as a trend toward brighter magnitudes with increasing apparent cluster radii.
\end{abstract}

Key words. Magellanic Clouds - galaxies: clusters: general - galaxies: evolution

\section{Introduction}

The proximity of the Magellanic Clouds (MCs) offers an excellent opportunity for studying their spatially resolved star formation (SF) histories. SF can be triggered by several mechanisms such as the self-induced gravitational collapse of a molecular cloud, tidal shocking, a turbulent interstellar medium, or cloudcloud collisions (e.g., McKee \& Ostriker 2007). The MCs and the Milky Way (MW) are interacting with each other. The formation of star clusters younger than $\lesssim 1 \mathrm{Gyr}$ in the MCs was probably triggered by interactions of the galaxies with each other and with the MW (e.g., Yoshizawa \& Noguchi 2003). Star clusters may be produced through strong shock compressions induced by close encounters of their host galaxies, which causes enhanced star formation. Conversely, the star formation rate again decreases once the galaxies recede from each other. Repeated encounters then lead to episodic cluster formation. In the MCs, a correlation between young star clusters and putative close encounters with each other and MW has been suggested by Girardi et al. (1995, G95), Pietrzynski \& Udalski (2000, PU00), and Chiosi et al. (2006, C06), among others.

* Full Tables 1 and 2 are only available in electronic form at the CDS via anonymous ftp to cdsarc.u-strasbg.fr $(130.79 .128 .5)$ or via

http://cdsweb.u-strasbg.fr/cgi-bin/qcat?J/A+A/517/A50
Strong tidal perturbations induced by the encounters could also have triggered the formation of clusters (e.g., Whitmore 1999) in the MCs. Possible orbits of the Small Magellanic Cloud (SMC), the Large Magellanic Cloud (LMC), and of the MW have been modeled by several authors (e.g., Bekki \& Chiba 2005). They find that it was difficult to keep the Clouds bound to each other for more than 1 Gyr in the past. The LMC and the SMC have been part of a triple system with the MW for at least 1 Gyr (e.g., Bekki \& Chiba 2005; Kallivayalil et al. 2006a,b). It is possible that the Clouds are not a bound system and that they are making their first passage close to the MW.

Interestingly, the cluster formation histories of the LMC and SMC show large differences. In the LMC, two main epochs of cluster formation (e.g., Bertelli et al. 1992) have been observed that are separated by an "age gap" of about 4-9 Gyr (e.g., Holtzman et al. 1999; Johnson et al. 1999; Harris \& Zaritsky 2001), in which no star clusters have formed. The two epochs of pronounced cluster formation occurred $>9$ Gyr ago and 3-4 Gyr ago. In the LMC, a few globular clusters are found that are as old as the oldest Galactic globulars (Olsen et al. 1998). During the past $\sim 4 \mathrm{Gyr}$, star clusters have been forming continuously until the present day. In contrast, the star clusters in the SMC cover a wide range of ages and have continued to form over at least the past $\sim 10.5$ Gyr (e.g., Glatt et al. 2008a,b; Parisi et al. 2009). Interestingly, the cluster formation history in the 
SMC appears to have started with a delay since the SMC formed its first and only globular cluster, NGC 121, 2-3 Gyr later than the LMC or the MW (Glatt et al. 2008a, and references therein). The LMC contains about $\sim 4200$ star clusters, while in the SMC $\sim 770$ star clusters have formed (and survived). The cluster census is probably still incomplete, missing small and faint clusters that are yet to be detected. Ongoing and prospective spacebased observations may further increase the number of known star clusters.

The most recent catalog that cross-correlates all known objects of the LMC, SMC, and the Magellanic Bridge region has been published by Bica et al. (2008) (B08). However, the cluster sample is still highly incomplete as pointed out by the authors. Only for a few clusters in B08's catalog, have ages been determined. For young SMC clusters, Pietrzynski \& Udalski (1999) (PU99) used isochrone fitting on data from the Optical Gravitational Lensing Experiment (OGLE II; Udalski et al. 1998) to determine ages for 93 clusters. Dieball et al. (2002) compiled ages for 306 binary cluster candidates in the LMC from a variety of literature sources ranging from multiwavelength integrated light studies to isochrone fitting to resolve clusters. Rafelski \& Zaritsky (2005) (RZ05) made use of integrated colors and derived ages for 200 clusters. C06 determined ages of 164 associations and 311 star clusters based on data from the OGLE using isochrone fitting. Their sample is the largest available catalog with SMC cluster ages. Ages for young LMC clusters have been provided by G95 based on integrated colors (624 objects) and by PU00 using isochrone fitting applied to OGLE-II data ( $\sim 600$ clusters).

Luminosities have been published for 204 SMC star clusters by RZ05 measuring integrated colors from the Magellanic Clouds Photometric Survey (MCPS). Bica et al. (1996) (B96) published integrated photometry of 624 LMC star clusters that was based on observations carried out at the 0.61-m telescope at CTIO in Chile and at the 2.15-m CASLEO telescope in Argentina.

In the present study we increase the number of age-dated young LMC and SMC star clusters and calculate $V$-band luminosities. We aim at improving the understanding of the cluster age distribution of these two irregular galaxies and present spatial distribution maps of the star clusters in both galaxies. To achieve this goal, we make use of ground-based data of the Magellanic Clouds Photometric Surveys (MCPSs) (Zaritsky et al. 2002, 2004). In the next section the observations and data reduction are described. In Sect. 3 the distances, reddenings, and metallicities of both the SMC and the LMC are given. In Sect. 4 the clusters' age distribution, spatial distribution, and dissolution effects are discussed, and in Sect. 5 the correlation between the cluster luminosities and age/radius is derived.

\section{Cluster section}

We made use of three catalogs in order to select clusters and to obtain color-magnitude diagrams (CMDs) of the young SMC and LMC star clusters (age $<1$ Gyr). The first catalog was published by B08 (see also Bica \& Schmitt 1995; Bica \& Dutra 2000) and includes cluster positions, angular sizes, and object classes for 17815 objects in the LMC, SMC, and Magellanic Bridge. This catalog combines and cross-identifies objects measured on the ESO/SERC R and J Sky Survey Schmidt films and other catalogs (e.g., Pietrzynski \& Udalski 1999).

The two other catalogs are from the MCPS presented by Zaritsky et al. $(2002,2004)$ containing $U, B, V$, and $I$ pointsource photometry of the central $18 \mathrm{deg}^{2}$ area of the SMC
(5 156057 stars) and of the central $64 \mathrm{deg}^{2}$ area of the LMC (24107014 stars). The data were obtained using the Las Campanas 1-m Swope telescope in Chile. The $V$ frame was used as reference, and only stars detected in both the $V$ and $B$ frame were retained. In both catalogs, the limiting magnitude of the photometry is $V \sim 24 \mathrm{mag}$. The photometry is highly incomplete below $U=21.5 \mathrm{mag}, B=23.5 \mathrm{mag}, V=23 \mathrm{mag}$, and $I=22 \mathrm{mag}$. This means that it is difficult to derive ages of star clusters older than $\sim 1$ Gyr because of the limited photometric depth of the MCPS, which does not resolve the main-sequence turnoff-points (MSTOs) of intermediate-age and older clusters.

Each object in B08's catalog is categorized by an object class. Obvious star clusters are indicated by a "C" and emissionless associations by an "A". The "CA" and "AC" indicate intermediate classes between these two types for which a classification was not clear, and "NC" refers to small HII regions with embedded star clusters, while "CN" are clusters that show traces of emission. The "DCN" refers to clusters that are decoupled from nebulae. For our study, only objects including a "C"'-classification were used (SMC: 765 objects; LMC: 4089 objects), because these objects are well-defined and not too extended so that cluster stars can be distinguished from the surrounding field stars. Star clusters that have very small radii or that are very sparse could not be age-dated (see Sect. 4). Our resulting catalogs contain ages of star clusters between $\sim 10 \mathrm{Myr}$ and 1 Gyr. Clusters whose appearance in the CMDs indicated an age over 1 Gyr were discarded from the sample, because the MSTOs were not resolved. Because we only age-dated objects including a "C" in the B08 classification, our samples only contain objects with a minimum age of $10 \mathrm{Myr}$, since very young objects are usually classified as associations or nebulae, so were excluded from our sample. For many intermediate-age and old SMC and LMC clusters, accurate ages have been determined elsewhere in the literature mostly based on HST data (e.g., Mighell et al. 1998; Olsen et al. 1998; Rich et al. 2000; Piatti et al. 2001; Glatt et al. 2008a,b).

\section{Metallicity, distance modulus, depth extent}

\section{$S M C$}

The latest low-resolution spectroscopic measurements of SMC star clusters by Parisi et al. (2009) shows that the young clusters with ages between $\sim 0.9$ and 2 Gyr have a spread in metallicity of 0.53 dex. The two youngest clusters in their sample, Lindsay 106 and Lindsay 108, both with ages around 0.9 Gyr, have mean metallicities of $[\mathrm{Fe} / \mathrm{H}]=-0.88$ and -1.05 , respectively. Studies based on the spectroscopy of six supergiants in the young cluster NGC 330 (age 20-25 Myr; Grebel et al. 1996) rendered a metallicity of $[\mathrm{Fe} / \mathrm{H}]=-0.69 \pm 0.11$ dex (e.g., Hill 1999), whereas Gonzalez \& Wallerstein (1999) find $[\mathrm{Fe} / \mathrm{H}]=$ $-0.94 \pm 0.02$ from high-resolution sprectra of seven supergiants in this cluster. It has been suggested repeatedly that NGC 330 is more metal-poor than the surrounding field star population (e.g., Grebel \& Richtler 1992; Gonzalez \& Wallerstein 1999). On the other hand, most field star studies agree on a value of $[\mathrm{Fe} / \mathrm{H}]=$ $-0.70 \pm 0.07$ dex (e.g., Hill et al. 1997; Venn 1999). This metallicity corresponds to an isochrone model of $Z=0.004$, which we used for the age determination. We do not consider a possible metallicity spread here.

A distance modulus of $(m-M)=18.90 \mathrm{mag}(\sim 60 \mathrm{kpc}$, e.g., Storm et al. 2004) was assumed for the SMC. There are no direct determinations of the cluster distances along the line of sight, but it is assumed that the SMC has a depth extent of up to $20 \mathrm{kpc}$ 
(Mathewson et al. 1988; Hatzidimitriou et al. 1993; Crowl et al. 2001; Lah et al. 2005; Glatt et al. 2008b). The clusters analyzed in our study lie in the central region of the SMC main body. Chiosi et al. (2006) find a variation in the distance modulus of $\sim 0.14$ mag assuming an elongation of $4 \mathrm{kpc}$. The resulting error in $\log ($ age $)$ is less than 0.05 .

\section{$L M C$}

Keller \& Wood (2006) studied 19 Cepheid variables in the LMC and derived a mean present-day metallicity of $[\mathrm{Fe} / \mathrm{H}]=-0.34 \pm$ 0.03 dex for this galaxy. This result agrees with previous determinations of the metal abundance within the young population of the LMC (e.g., Luck et al. 1998; Romaniello et al. 2005) also using Cepheid variables. This metallicity matches the isochrone models of $Z=0.008$ best, which were used for the age determination.

For the LMC, a distance modulus of $(m-M)=18.50 \mathrm{mag}$ ( $\sim 50 \mathrm{kpc}$; e.g., Alves 2004) was assumed. There are indications that the LMC bar may be offset from the plane of the disk by about 2 kpc (e.g., Zhao \& Evans 2000; Subramaniam $\&$ Subramanian 2009). This leads to a variation in the distance modulus of $\sim 0.08 \mathrm{mag}$. The resulting error in $\log ($ age $)$ is less than 0.05 .

\section{Cluster age distribution}

The cluster age distribution of the LMC and the SMC was obtained by first matching the clusters' central positions adopted from B08 with the MCPS (Zaritsky et al. 2002, 2004). From the apparent major and minor axis adopted by B08 we computed the mean apparent diameter

$D_{\text {app }}=(a+b) / 2$

for each object (e.g., the SMC cluster NGC 376 in Fig. 1). All stars within these diameters are considered cluster members. Four CMDs were plotted for each object: two $V$ vs. $B-V$ CMDs and two $V$ vs. $V-I$ CMDs showing the cluster and the surrounding field.

We fitted the CMDs with two different isochrone models: Padova isochrones (Girardi et al. 1995) and Geneva isochrones (Lejeune \& Schaerer 2001). Both the Padova and the Geneva isochrone grids have an age resolution of $\log (t)=0.05$. The Dartmouth isochrone models have a youngest age of $250 \mathrm{Myr}$ (Dotter et al. 2007), which is too old for many of the star clusters in our sample, so we did not use these models. To fit the isochrones, a constant distance modulus and metallicity were used. The best-fit isochrone was then found by using different combinations of reddening and age, which were derived for each cluster by visual inspection. Human judgement is needed in particular to decide about including luminous supergiants in the fit, since the apparent main-sequence turnoff of sparse, young clusters is subject to pronounced statistical fluctuations (e.g., Lançon \& Mouhcine 2000). The Geneva isochrone models with $Z=0.004$ deviate from the Padova isochrones for ages younger than $\log ($ age $)=6.9$ by about $\log ($ age $)=-0.1$. The Geneva models with $Z=0.008$ deviate by about $\log ($ age $)=-0.1$ from the Padova isochrones for ages younger than $\log ($ age $)=8.00$. Our catalogs list the ages determined with the Padova isochrones.

Many star clusters in the present samples are located in the main body of the SMC and along the LMC bar, both of which are highly crowded. Therefore, field star contamination severely

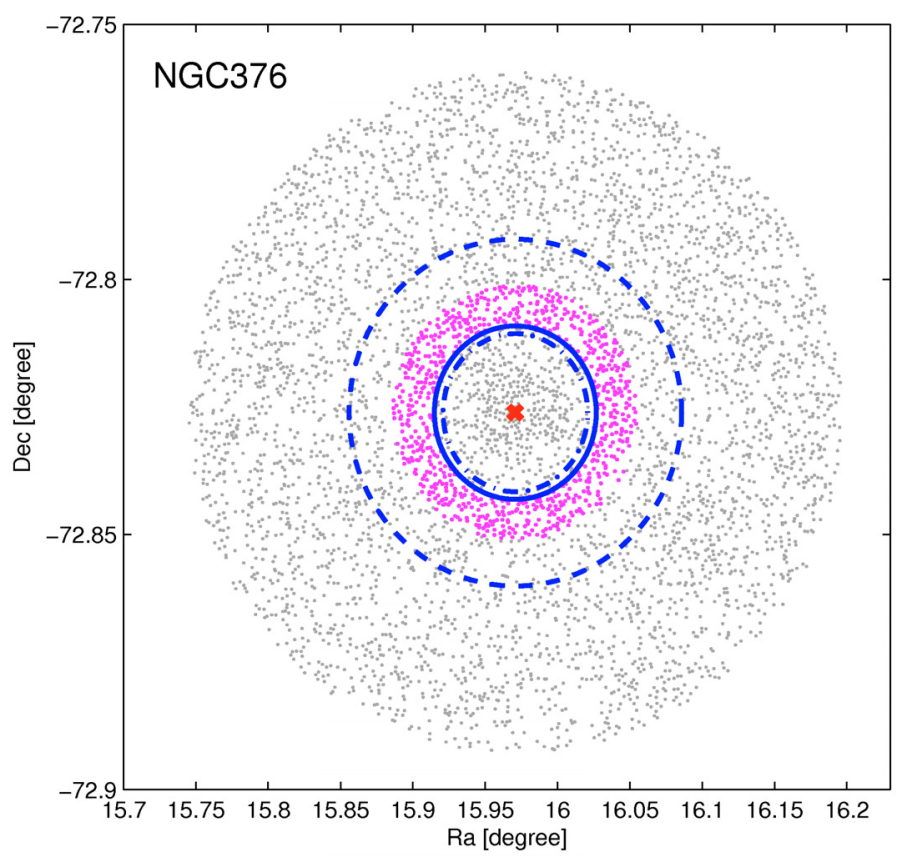

Fig. 1. Spatial distribution of the SMC star cluster NGC 376 (MCPS, $V<20.5 \mathrm{mag}$ ). The red cross denotes the cluster center (B08). The innermost, blue dash-dotted line represents $r_{\text {app }}=0.9 \operatorname{arcmin}$ (B08). The solid blue line shows a circle 1 arcmin away from the cluster center, while the dashed blue line lies 2 arcmin away. Stars lying between these two circles were plotted in the lower panels of Figs. 2 and 3 to visualize the field star populations. The stars plotted as magenta dots are located between $r_{\text {app }}+0.1$ and $r_{\text {app }}+0.6$. They correspond to the magenta dots shown in the upper panels of Figs. 2 and 3.

influences the age determination. The field population was sampled within an annulus between 1 and 2 arcmin around the cluster centers outside the apparent cluster radii (e.g., lower panels of Figs. 2 and 3). For clusters with larger apparent radii than 1 arcmin, the field population was sampled within an annulus between 2 and 3 arcmin. The selected stars lying within these annuli were plotted on top of the cluster CMDs to illustrate the location of the SMC field stars. The accuracy of the inferred ages depends on the number of cluster member stars and on the density of the surrounding field. Many star clusters in the sample could not be age-dated, because they are too sparse or they have very small radii, which include too few stars against the SMC field background for a reliable isochrone fit. In the densest SMC regions, the cluster membership determination on the basis of photometric information is difficult, making the here derived ages uncertain.

Two examples are shown in Figs. 2 and 3. For the SMC cluster NGC 376, we derived an age of $\sim 30 \mathrm{Myr}$ and $E_{B-V}=0.08$. The LMC cluster SL410 is a slightly older star cluster for which we determined an age $\sim 110 \mathrm{Myr}$ and $E_{B-V}=0.07$. In the respective upper panels, stars that were selected within the cluster radii adopted from B08 are displayed. Field stars were selected within a concentric 0.5 arcmin wide annulus located 0.1 armin away from the clusters apparent radii to estimate the field star contamination. In the lower panels, field stars selected within an annulus between 1 and 2 arcmin radius around the cluster centers are shown.

Young cluster ages derived from isochrone fitting are not severely affected by the discreteness of isochrones due to the logarithmic age-steps of the applied isochrone models, which produce a fairly dense grid for small ages. The 


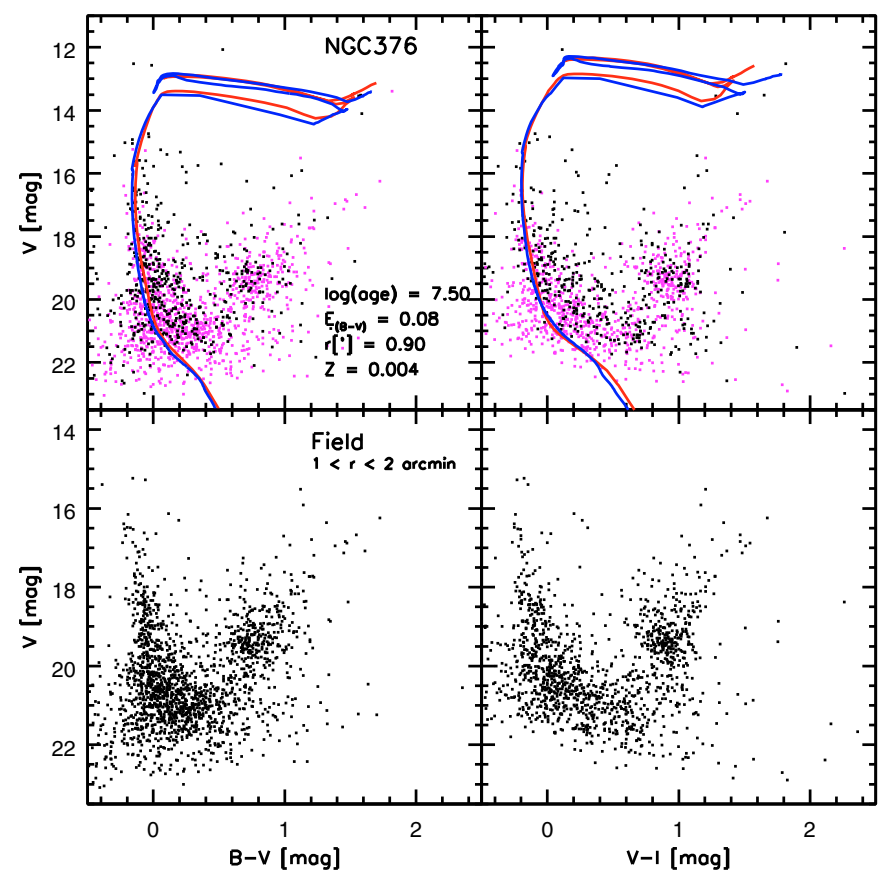

Fig. 2. Color-magnitude diagrams of the SMC star cluster NGC 376. In the upper panel, $V$ vs. $B-V$ and $V$ vs. $V-I C M D s$ including stars within a radius of 0.90 arcmin (B08) are shown, and in the lower panel field stars within a concentric annulus between 1 and 2 arcmin are displayed. The blue solid line shows the best-fitting Geneva (Lejeune \& Schaerer 2001) isochrone. The red solid line is the best-fitting Padova (Girardi et al. 1995) isochrone. The magenta dots were selected in a $0.50 \mathrm{arcmin}$ concentric annulus that is located 0.1 arcmin away from the cluster apparent radius. They indicate the location of the SMC field stars in the CMDs. The fitted parameters are noted in the figure.

interstellar extinction, however, can have a severe impact on the determination of the cluster ages. We compared the reddenings found by isochrone fitting to the reddenings adopted from PU99. The standard deviation is approximately $\sigma E_{(B-V)}=0.03$.

The uncertainty in the present-day metallicity of the SMC is mentioned in Sect. 3. Therefore, for some clusters, the applied isochrone metallicity of $Z=0.004$ might not be the best choice. However, the attempt to fit the clusters with a different metallicity (e.g., $Z=0.008$ ) provided fits of lower quality (age difference of $\sim \log ($ age $)=0.2$ ). Therefore, the uncertainties of the age determinations are partly a function of interstellar extinction, partly a function of the age itself in the sense that older clusters are more difficult to identify, and partly a function of the cluster density.

An uncertainty that cannot be handled with our data occurs when the clusters are all inside large groups of associations having small separations along the line of sight or when they are very sparse. Sparse objects usually do not have a well-defined main sequence turn-off. On one hand, supergiants with their short lifetimes (typically $\sim 20 \mathrm{Myr}$ ) are well-suited as age tracers if they show a concentration toward the cluster center. We therefore require our "best" isochrones to reproduce the location to the supergiants in the CMDs and use that as a primary criterion for age determinations. The reddenings, on the other hand, are based on the perceived locus of the blue envelope of the main sequence. The determination of cluster membership for individual stars is a difficult matter (based on only photometric information) and further investigation with, e.g., the Hubble Space Telescope or studies based on spectroscopy are necessary. A clear separation between clusters and their surroundings

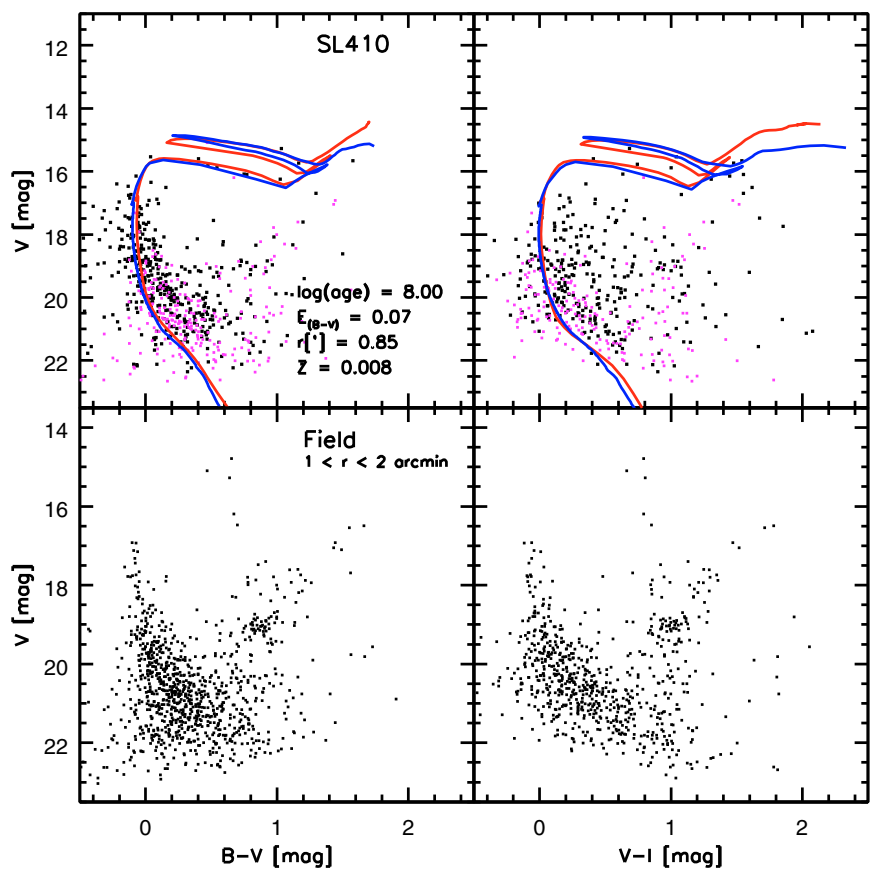

Fig. 3. As in Fig. 2 but for the LMC cluster SL410.

becomes particularly difficult when they are embedded in associations of a very similar age.

For the SMC and the LMC, ages of 324 clusters and 1193 clusters, respectively, have been determined in our study. The full tables will be made available electronically, but illustrative excerpts are shown in Tables 1 and 2. Column 1 gives the cluster identification number. The reddening parameter $E_{B-V}$ is listed in Col. 2 and the apparent radii in Col. 3. The derived ages are shown in Col. 4, while Col. 5 gives the degree of reliability of our age measurements. Class 1 indicates having errors $\Delta \sigma_{\log (\text { age })}<0.3$, class 2 indicates objects having errors $0.3 \leqslant \sigma_{\log (\text { age })}<0.5$, and class 3 indicates objects having errors $\sigma_{\log (\text { age })} \geqslant 0.5$, adopting the same notation as used by $\mathrm{C} 06$. Columns 6 and 7 give the right ascension and the declination, respectively. The calculated total $V$ band magnitudes (see Sect. 5) are listed in Col. 8 and finally, the cross-identifications with other catalogs are given in Col. 9.

\subsection{Comparison of our age determination with previous studies}

C06 uses data from OGLE II (Udalski et al. 1998) for the SMC disk and data obtained at the ESO $2.2 \mathrm{~m}$ telescope for the region around NGC 269 that is located at the border of the supershell 37 A to derive ages of 461 SMC clusters and associations. The ages were derived using Padova isochrones (Girardi et al. 2002). A distance modulus of $(m-M)=18.9$ was assumed and the reddening was derived by main-sequence fitting. A mean SMC metallicity of $Z=0.008$ was assumed (in the present study we used $Z=0.004$ ) as found spectroscopically for young stars by Pagel \& Tautvaišienè (1999). An upper age limit was set by the limiting magnitude of the available photometry indicating that clusters that have an MSTO fainter than $V=20$ mag in the OGLE II field could not be age-dated. The C06 study was restricted to clusters younger than $1 \mathrm{Gyr}$. They derived ages within an age range of $4 \mathrm{Myr}$ and $1 \mathrm{Gyr}$ for clusters covering an area of $2.4 \mathrm{deg}^{2}$ of the SMC main body, and 136 of their clusters are also included in our study. 
K. Glatt et al.: Ages and luminosities of young SMC/LMC star clusters and the recent star formation history of the Clouds

Table 1. SMC cluster catalog including determined ages and $V$-band luminosities (full table available at the CDS).

\begin{tabular}{ccccccccl}
\hline \hline ID & $\begin{array}{c}E_{B-V} \\
\text { mag }\end{array}$ & $\begin{array}{c}R_{\text {app }}{ }^{a} \\
\text { arcmin }\end{array}$ & $\begin{array}{c}\log (\mathrm{age}) \\
\mathrm{yr}\end{array}$ & $\sigma_{t}$ & $\begin{array}{c}\text { Ra } \\
\mathrm{J} 2000.0\end{array}$ & $\begin{array}{c}\text { Dec } \\
\mathrm{J} 2000.0\end{array}$ & $\begin{array}{c}V^{b} \\
\text { mag }\end{array}$ & Cross - ID \\
\hline SMC 0017 & 0.02 & 0.25 & 9.00 & 2 & $0: 28: 31$ & $-73: 00: 49$ & $16.95 \pm 0.14$ & BS2 \\
SMC 0018 & 0.01 & 0.60 & 8.70 & 1 & $0: 30: 00$ & $-73: 22: 45$ & $14.10 \pm 0.13$ & K9, L13 \\
SMC 0023 & 0.01 & 0.60 & 9.10 & 3 & $0: 32: 41$ & $-72: 34: 53$ & $14.77 \pm 0.15$ & L14 \\
SMC 0026 & 0.03 & 1.50 & 8.85 & 3 & $0: 32: 56$ & $-73: 06: 58$ & $12.51 \pm 0.12$ & NGC 152, K10, L15, ESO 028SC24 \\
\hline
\end{tabular}

Notes. ${ }^{(a)}$ The apparent radii (Eq. (1)) as adopted from B08; ${ }^{(b)}$ the $V$ magnitudes correspond to the total luminosity of all stars within $R_{\text {app }}$ (see Sect. 5).

Table 2. LMC cluster catalog including determined ages and $V$-band luminosities (full table available at the CDS).

\begin{tabular}{ccccccccl}
\hline \hline ID & $\begin{array}{c}E_{B-V} \\
\text { mag }\end{array}$ & $\begin{array}{c}R_{\text {app }}{ }^{a} \\
\text { arcmin }\end{array}$ & $\begin{array}{c}\log (\text { age }) \\
\text { yr }\end{array}$ & $\sigma_{t}$ & $\begin{array}{c}\text { Ra } \\
\text { J2000.0 }\end{array}$ & $\begin{array}{c}\text { Dec } \\
\text { J2000.0 }\end{array}$ & $\begin{array}{c}V^{b} \\
\text { mag }\end{array}$ & Cross - ID \\
\hline LMC 0020 & 0.02 & 0.75 & 8.80 & 1 & $4: 37: 51$ & $-69: 01: 45$ & $13.89 \pm 0.14$ & SL8, LW13, KMHK21 \\
LMC 0021 & 0.08 & 0.29 & 8.00 & 1 & $4: 38: 07$ & $-68: 46: 39$ & $14.29 \pm 0.14$ & NGC 1649, ESO055SC31, KMHK22 \\
LMC 0022 & 0.08 & 0.75 & 8.50 & 1 & $4: 38: 22$ & $-68: 40: 21$ & $13.11 \pm 0.14$ & SL14, LW21, KMHK28 \\
LMC 0030 & 0.02 & 0.55 & 8.30 & 2 & $4: 40: 28$ & $-69: 38: 57$ & $13.73 \pm 0.12$ & SL15, LW23, KMHK29 \\
\hline
\end{tabular}

Notes. ${ }^{(a)}$ The apparent radii (Eq. (1)) as adopted from B08; ${ }^{(b)}$ the $V$ magnitudes correspond to the total luminosity of all stars within $R_{\text {app }}$ (see Sect. 5).

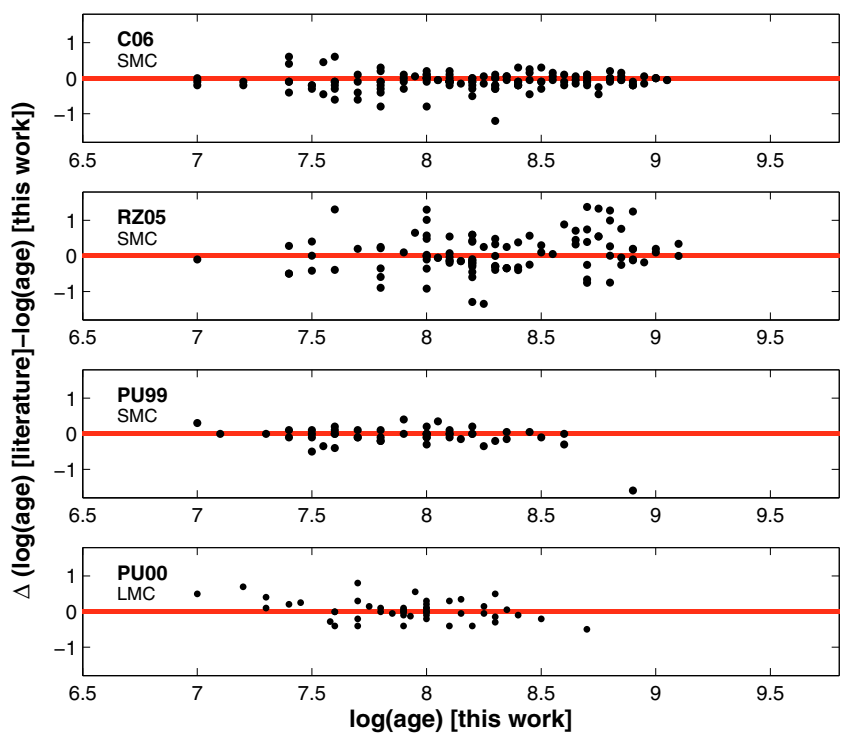

Fig. 4. Cluster ages derived in this work are compared with the SMC cluster ages by C06 (first panel), RZ05 (second panel), and PU99 (third panel), and with the LMC cluster ages by PU00 (fourth panel) for the clusters in common.

In the first panel of Fig. 4 cluster ages determined in our work are compared to ages presented by C06. The ages derived in our work tend to be $\sim 0.2-0.3$ in $\log$ (age) older than the ages by $\mathrm{C} 06$, an offset we anticipated above. The main reason for the difference is probably the different metallicity of the applied isochrone models since we tested for a subset of CMDs. For those clusters with the most significant age deviation, large age uncertainties are stated in both their and our works (e.g. BS271, BS272, B114). The dispersion about the zero line for the sample is $\sigma_{\log (\text { age })}=0.13$.

RZ05 derived ages of 204 SMC star clusters using integrated colors, of which 112 clusters are included in our sample. The second panel of Fig. 4 shows the comparison between our ages and ages adopted by RZ05. The largest deviations are found at the ends of the upper and the lower age limits. A few clusters having ages younger than $25 \mathrm{Myr}$ and older than 1 Gyr show an age deviation of up to $1 \mathrm{Gyr}$. Stochastic effects on the number of bright stars, uncertainties on the metallicity, dubious cluster membership, and on the adopted stellar models may contribute to the large uncertainties in the conclusion of the cluster age. RZ05 used a metallicity of $Z=0.004$. Those clusters with the largest age differences are mostly very sparse objects or objects to which very young isochrones were fitted based on only 2-3 bright stars. Large uncertainties for the oldest clusters are caused by large field contamination and unresolved mainsequence turnoff points. The dispersion about the zero line for the sample is $\sigma_{\log (\text { age })}=0.3$.

PU99 determined ages for 93 SMC star clusters from the OGLE catalog (Pietrzynski et al. 1998), of which 51 are included in our sample. They fitted isochrones from the library of Bertelli et al. (1994) applying a distance modulus of $(m-M)_{0}=18.65$ (Udalski 1998) and a metallicity of $Z=0.004$. In their study, they derived cluster ages within an age range of $10 \mathrm{Myr}$ and 1 Gyr. In the third panel of Fig. 4 we compare ages presented by PU99 to our study. The ages are in very good agreement and the dispersion about the zero line is $\sigma_{\log (\text { age })}=0.17$. This result may seem surprising considering the difference in the applied distance moduli of 0.25 . However, fitting isochrones to the same CMD using both values for the distance moduli leads to age differences for these young clusters of no more than $0.1-0.15$ in $\log$ (age).

Ages of about 600 LMC star clusters were determined using OGLE II data (Udalski et al. 1998) by PU00. The ages were derived fitting isochrones of Bertelli et al. (1994). A distance modulus of 18.24 mag and a metallicity of $Z=0.008$ were assumed. The reddenings were adopted from Udalski et al. (1999). Clusters older than $\sim 1.2$ Gyr could not be reliably age-dated, because the main-sequence turnoff points are located close to the limit of the OGLE II photometry ( $V \approx 21.5 \mathrm{mag}$ ). 49 clusters are in common with our cluster sample. In the fourth panel of Fig. 4 we compare the derived ages found in this study with PU00. The dispersion about the 1:1 agreement for the sample is $\sigma_{\log (\text { age })}=0.15$. Again the good agreement is surprising, considering the difference of the applied distance moduli of 0.26 . As for the SMC, the age difference for these young clusters is 

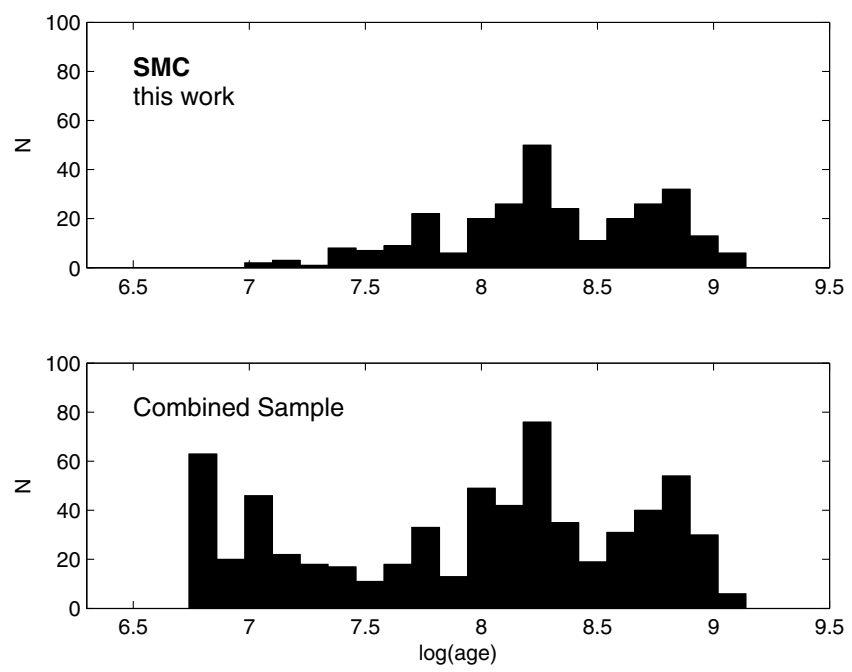

Fig. 5. Cluster age distribution of the SMC. The age distribution derived in this study (upper panel) and the combined samples (lower panel) of this study and C06 are shown (in total 821 clusters). Only the most reliable ages were considered for this plot (classes 1 and 2 in Table 1).
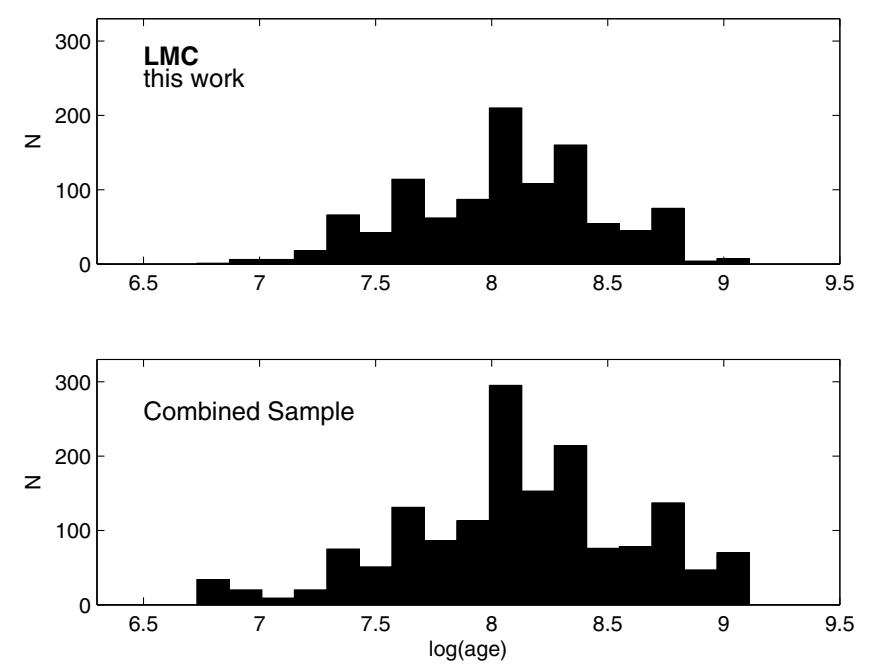

Fig. 6. Cluster age distribution of the LMC. The age distribution derived in this study (upper panel) and the combined samples (lower panel) of this study and PU00 are shown (in total 1745 clusters). Only the most reliable ages were considered for this plot (classes 1 and 2 in Table 2). no more than $0.1-0.15$ in $\log ($ age $)$ fitting two isochrones to the same CMD using both distance modulus values.

G95 used integrated colors to determine the $\mathrm{S}$ parameter for all 624 LMC objects listed in the catalog of Bica et al. (1996). The $\mathrm{S}$ parameter is an age indicator based on integrated $U-B$ and $B-V$ colors (see also Elson \& Fall 1985). Unfortunately, a direct comparison of our data is impossible because the cluster ages of G95 are not tabulated. Grebel et al. (1999) fitted isochrones to data from earlier versions of the MCPS (Zaritsky et al. 1997) and OGLE (Udalski et al. 1998) and transformed G95's S parameter into ages. The authors found good overall agreement in the age distribution.

All in all, the scatter of $\sim 0.2$ in age derived by other groups and in this work is in good agreement with the typical age uncertainties derived in either study.

\subsection{Age distribution}

Uncertainties aside, the age distribution of star clusters is influenced by two effects (Boutloukos \& Lamers 2003). The first is cluster fading that occurs through stellar evolution when the clusters get fainter with time. Therefore, the number of observed star clusters appears to decrease with increasing age for a given magnitude limit since sparse, faint clusters become increasingly harder to detect. A second effect acting upon gas-free clusters is caused by the combined effects of 2-body relaxation and external tidal stripping: this is the so-called secular evolution (Baumgardt \& Makino 2003). A third effect altering the initial age distribution at the lower end is infant mortality caused by gas expulsion. In the MCs the phase of infant mortality lasts up to $\approx 40 \mathrm{Myr}$ (de Grijs \& Goodwin 2009).

The cluster age distributions of the SMC and the LMC are shown in Figs. 5 and 6. The upper panels of these figures display only cluster ages determined in our study and in the lower panels the combination of this study with C06 (SMC) and PU00 (LMC) samples. For the clusters in common with our work, we used ages derived in this study. Because we only age-dated objects that included a " $\mathrm{C}$ " in the B08 classification, our samples contain only a few objects younger than $\sim 20 \mathrm{Myr}$, since very young objects are usually classified as associations or nebulae, so were excluded from our sample.

The age distribution of the SMC star clusters derived in our study shows a first peak of enhanced cluster formation around $160 \mathrm{Myr}$ and a second one around $630 \mathrm{Myr}$. Only clusters with an age uncertainty smaller than class 3 were used. C06 found two main episodes of enhanced cluster formation between 5-15 Myr and at 90 Myr. Their cluster sample contains ages for 164 associations, which is the reason their sample reaches down to ages of $\sim 3$ Myr. In the combined sample three episodes of enhanced cluster formation are detectable, the youngest around 6.5 Myr, followed by a second peak around $160 \mathrm{Myr}$, and a third around 630 Myr. The analysis in the age range of the oldest peak is limited by the depth of the photometry, while the youngest peak mostly comes from the associations adopted from C06. The difference between the oldest peak found in our study and by $\mathrm{C} 06$ lies within the given uncertainties for the cluster ages $\left(\sigma_{\log (\text { age })}=0.3\right)$.

For the LMC, PU00 detected three periods of enhanced cluster formation at about $7 \mathrm{Myr}, 125 \mathrm{Myr}$, and $800 \mathrm{Myr}$, which are similar to the findings of G95. No associations and nebulae were considered in the study of PU00 so that the age distribution of the combined LMC sample does not reach as young an age as the combined SMC sample. The youngest clusters in PU00's sample have ages of $\sim 5 \mathrm{Myr}$, but come with high age uncertainties as stated by the authors. The age determination of the oldest clusters in our sample with ages close to $1 \mathrm{Gyr}$ is highly uncertain (class 3). Clusters with uncertainties below class 3 were discarded making the oldest peak very weak. In the combined sample an increased number of clusters appears at $~ 9$ Myr and $630 \mathrm{Myr}$, which is in very good agreement with G95 and P00. The most enhanced peak occurs at about $125 \mathrm{Myr}$.

In both the SMC and the LMC, we find evidence of periods of enhanced cluster formation, which appear to have occurred during the same periods. The peaks at $\sim 125$ and $160 \mathrm{Myr}$, respectively, are very pronounced in both galaxies and are probably correlated. The difference between the peaks is within the given uncertainties for the cluster ages (difference in $\log ($ age $)=0.1$. Model calculations performed by e.g., 
K. Glatt et al.: Ages and luminosities of young SMC/LMC star clusters and the recent star formation history of the Clouds

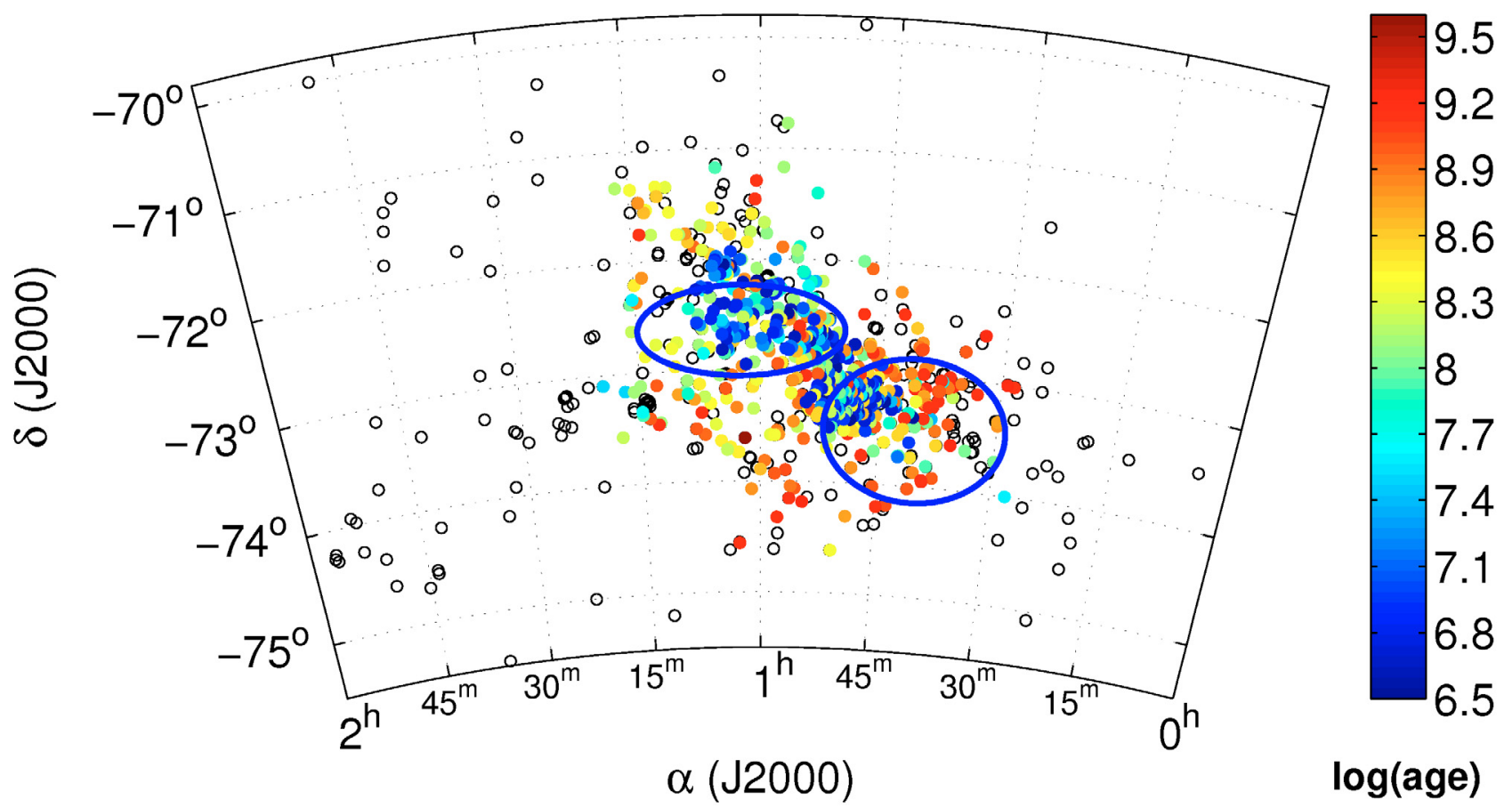

Fig. 7. Spatial distribution of the combined sample of this work and C06 of the young SMC star clusters. The two blue ellipses indicate the location of the HI super-shells $304 \mathrm{~A}$ (middle) and $37 \mathrm{~A}$ (right) (Stanimirovic et al. 1999). The open circles indicate all objects from B08 including a "C"-classification (in total 765 objects). North is up, west to the right.

Bekki \& Chiba (2005) and Kallivayalil et al. (2006a,b) show that the MW, the LMC, and the SMC have only interacted long enough to produce the Magellanic Stream. According to these models, the last close encounter between SMC and LMC occurred about 100-200 Myr ago. The SMC star formation rate increases, if the LMC orbit leads to a close encounter with the SMC and vice versa. The star formation rate decreases again when the LMC recedes from SMC, thus leading to episodic cluster formation. This increase in cluster formation some 100-200 Myr ago may therefore have been triggered by a tidal interaction with the neighboring galaxy (e.g., Gardiner \& Noguchi 1996; Bekki \& Chiba 2005; Kallivayalil et al. 2006a,b). The youngest peaks, however, might have another origin. Probably they are caused by associations, which have not yet dissolved. High velocity cloud-cloud collisions are another trigger mechanism of cluster formation (Zhang et al. 2001; Bekki et al. 2004). These collisions are particularly effective during galaxy interactions and mergers. High-speed motions may produce a high-pressure environment that in turn can trigger turbulences or shocks (Elmegreen \& Efremov 1997). Stellar winds and supernova explosions can also trigger star formation through compression by turbulent motions (Larson 1993).

\subsection{Spatial age distribution}

\section{$\mathrm{SMC}$}

The spatial distribution of the star clusters in Fig. 7 clearly shows that the youngest clusters are located in the two large HI supershells $37 \mathrm{~A}$ and 304 A (Stanimirovic et al. 1999). These features are believed to have their origin in the evolution of an $\mathrm{OB}$ association in which the most massive stars become supernovae and/or where stellar winds lead to a runaway expansion and the formation of a supergiant shell (e.g., Bruhweiler et al. 1980; Tomisaka et al. 1981; Elmegreen \& Chiang 1982). Most of the shells and supershells are associated with young objects (McCray \& Kafatos 1987). Inside the shells, second-generation star clusters may form from supernova explosions in the first-generation clusters.

Our (or the C06) data do not cover the western side of supershell $37 \mathrm{~A}$, but most of the remaining objects are age-dated. We confirm the findings of C06 about the discontinuity in the spatial distribution of younger clusters in the supershells $37 \mathrm{~A}$ and $304 \mathrm{~A}$. That is, the youngest objects are found toward the eastern rim of the shell (37A) where gas and dust are located (Staveley-Smith et al. 1997; Stanimirovic et al. 1999) and toward regions luminous in $\mathrm{H} \alpha$. Such $\mathrm{HII}$ regions visible in $\mathrm{H} \alpha$ indicate the presence of massive stars (e.g., OB-stars, supergiants, luminous blue variable stars), supernova remnants, or diffuse ionized nebulae. The youngest objects of the shell 304 A are located toward its northern and western rims (toward $37 \mathrm{~A}$ ) and also in the central part. The compression of gas due to shell-shell interactions related to the expansion of the shells might have triggered the cluster formation at the opposing rims, as well as in the intershell region. $\mathrm{H} \alpha$ is concentrated in the northern part of $304 \mathrm{~A}$ where we also find the youngest objects.

In Fig. 8 four snapshots are shown displaying the spatial distribution of star clusters within different age ranges. In the first only clusters younger than $16 \mathrm{Myr}$ are shown. The clusters are distributed along the SMC bar in an elongated but narrow area. The two supershells and the intershell region are clearly visible in this plot. Clusters with ages between $16 \mathrm{Myr}$ and $63 \mathrm{Myr}$ are also concentrated along the bar, but there are outliers found toward the SMC wing or slightly offset of the bar (second snapshot). The supershell $304 \mathrm{~A}$ is highly populated by clusters of this age range. Widely spread over the entire SMC main body are clusters with ages between $63 \mathrm{Myr}$ and $315 \mathrm{Myr}$ (third snapshot). Moreover, the northern part of the SMC body is covered 
age $<16 \mathrm{Myr}$

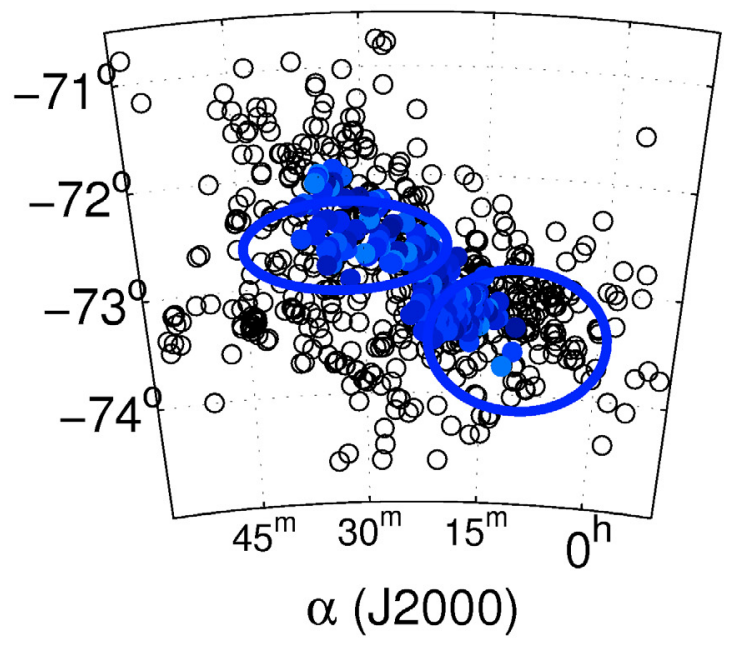

$63 \mathrm{Myr}<=$ age $<316 \mathrm{Myr}$

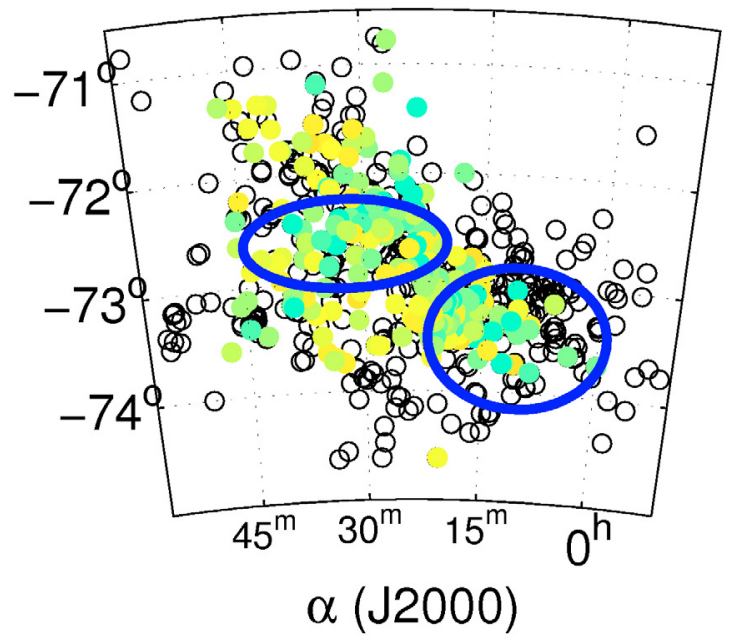

16 Myr <= age $<63$ Myr
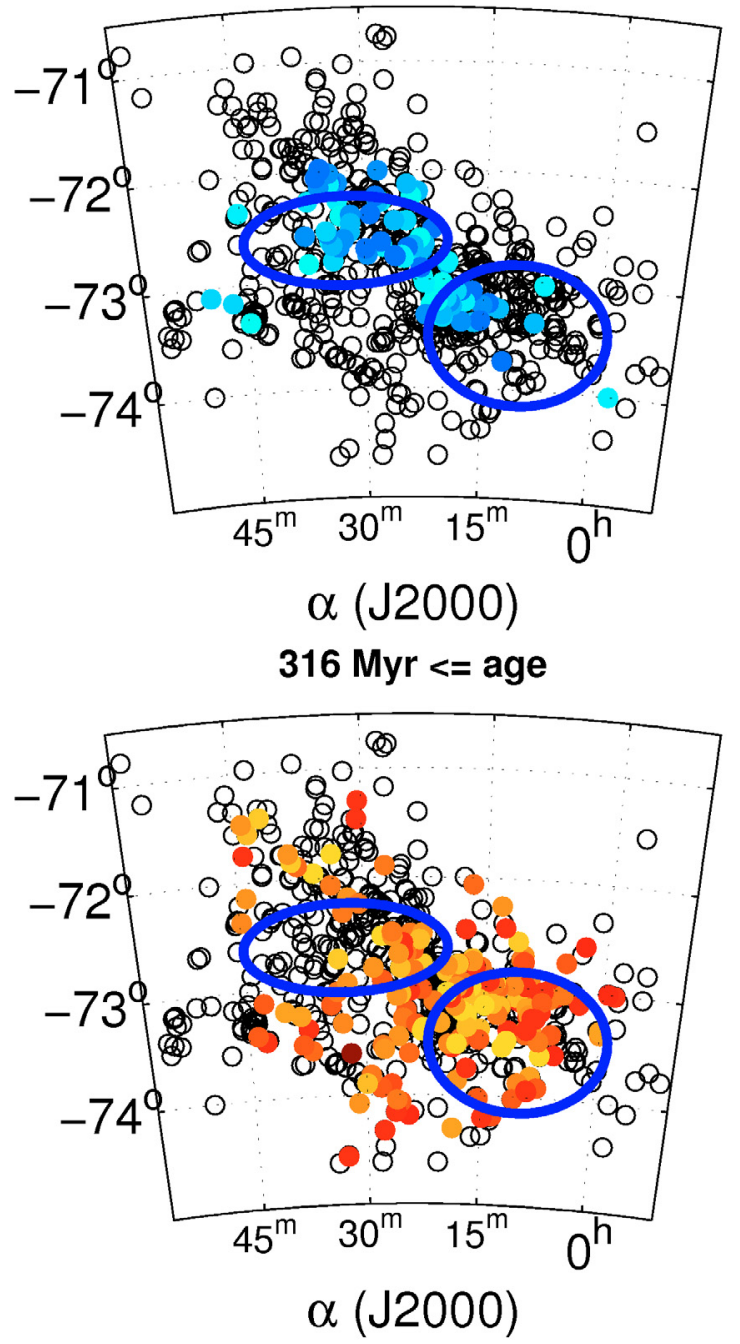

Fig. 8. Spatial distribution of SMC clusters younger than $1 \mathrm{Gyr}$ in different age bins.

with clusters in this age range. Finally, the last snapshot displays clusters older than $315 \mathrm{Myr}$ (up to $\sim 1 \mathrm{Gyr}$ ). These clusters mainly populate the western part of the SMC main body, and only a few are found in the north or in the east. Supershell 304 A contains fewer of these older objects than $37 \mathrm{~A}$, and they are mainly located at the western rim of the shell, while they are widely distributed in $37 \mathrm{~A}$. Generally, the cluster distribution in $304 \mathrm{~A}$ indicates a continuous cluster formation from a few Myr to $1 \mathrm{Gyr}$.

According to the standard model of shell formation (McCray \& Kafatos 1987), the older objects are supposed to be found in the center of the shell, while younger objects are distributed around the edge. As mentioned above, the younger objects in $37 \mathrm{~A}$ and $304 \mathrm{~A}$ are clustered toward the eastern and the western rims of the shells, respectively. Shell interaction due to collisions following shell expansion has probably triggered the cluster formation in the intershell region, as well as at the two opposing rims. The reason might be that the shell is surrounded by an interstellar medium with differing densities. Shell expansion into higher density regions leads to slower expansion, because the corresponding strong gas compression leads to enhanced star formation in that region. On the other hand, if the shell expands into a region with lower density, the star formation rate will be lower since there is less compression and less material to be swept up.

We confirm C06's finding that the location of the young SMC clusters is correlated with HI intensities, which decrease with increasing age. Mizuno et al. (2001a) find that young emission objects are positionally well correlated with $\mathrm{CO}$ clouds, while emissionless objects of ages 6-100 Myr do not show any correlation to the $\mathrm{CO}$ clouds. Emission objects were excluded from our sample, so we cannot investigate their possible association with $\mathrm{CO}$ clouds.

\section{LMC}

The youngest star clusters are found mostly in the LMC HI supergiant shells (SGSs) 30 Doradus, SGS 7 (LMC 5), SGS 11 (LMC 4), SGS 12 (LMC 3), and SGS 19 (LMC 2) and in the 103 giant shells published by Kim et al. $(1999,2003)$ (see also Davies et al. 1976; Meaburn 1980). The authors classify the shells into three categories; the single SGSs are simple expanding stalled shells, complex SGSs consist of smaller interlocked shells, and their rims often contain smaller shells (SGS 4, 9, 10, $12,17,18,20)$, and propagated $S G S s$ are surrounded by another SGS (SGS 11, 19). In most of the complex SGSs (see Fig. 9) 
K. Glatt et al.: Ages and luminosities of young SMC/LMC star clusters and the recent star formation history of the Clouds
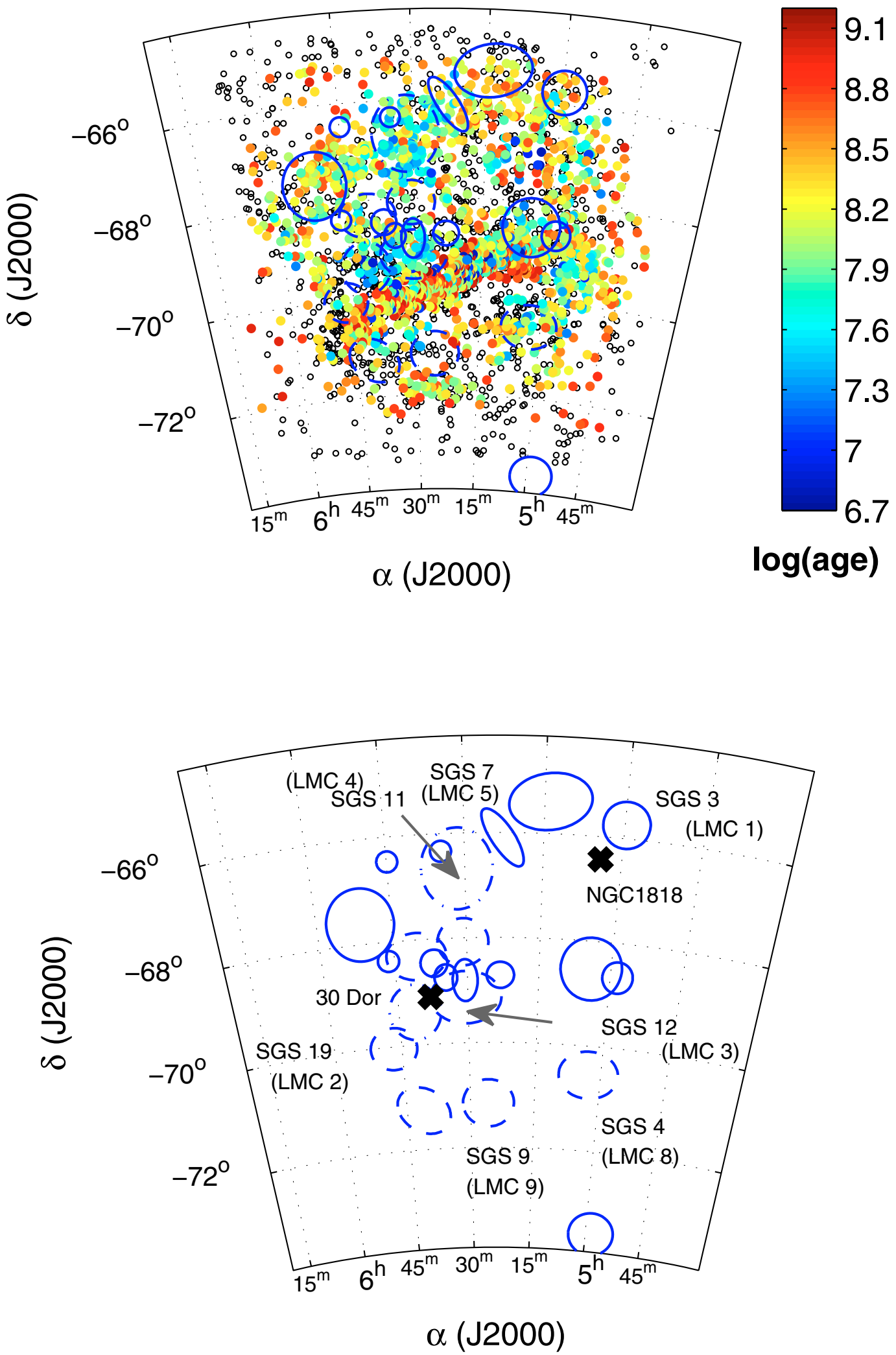

Fig. 9. Spatial distribution of the combined sample of this work and PU00 of the young LMC star clusters. The blue ellipses indicate the location of the 23 HI supershells (single SGSs (solid), complex SGSs (dashed), propagated SGSs (dash-dotted) Kim et al. 1999, 2003). Marked are all shells discussed in the text, SGS 3 (LMC 1), SGS 4 (LMC 8), SGS 7 (LMC 5), SGS 9 (LMC 9), SGS 11 (LMC 4), SGS 12 (LMC 3), and SGS 19 (LMC 2). The open circles indicate all objects from B08 including a "C"-classification (in total 4089 objects). The black crosses denote the locations of 30 Doradus and NGC 1818 (from left to right). North is up, west to the right.

star clusters are located along the rims and lack the youngest clusters. The smaller shells along their rims might indicate that triggered star formation has occurred recently. The propagated SGSs may have formed by sequential star formation. 
de Boer et al. (1998) proposed a scenario in which star formation in the LMC is triggered by the gas being compressed at the leading edge due to the bow shocks as the LMC moves through the gaseous Galactic halo around the MW. The bow shock compresses large areas, which leads to star formation on a large scale. Due to the LMC's rotation, the material at the leading edge will move away clockwise and distribute itself around the LMC's rim. In this scenario one would expect to find a progression in the age of star clusters along the direction of the rotation (in Fig. 9 the galaxy rotates clockwise). The LMC disk shows solid-body rotation and has an approximate full rotation velocity for a small inclination of $\sim 150 \mathrm{~km} \mathrm{~s}^{-1}$ at $1.5 \mathrm{kpc}$ (de Boer et al. 1998). The time of one full rotation period is $\sim 250 \mathrm{Myr}$. If present, the progression of age along the galaxy's rim should be detectable. The youngest shell structures lie in the proximity of 30 Doradus, the largest star-forming region of the LMC, and 30 Doradus lies close to the leading edge of the LMC. It is the largest star-forming region in the galaxy and may very well be related to the bow shock.

Grebel \& Brandner (1998) used Cepheids and other supergiant stars to study the recent star formation history of the LMC. They found that the majority of objects younger than $30 \mathrm{Myr}$ are concentrated on the southeastern border, while others are widely distributed across the entire disk which cannot be explained with the bow-shock star formation model. A progression of age was found in several giant shells along the LMC rim moving from the southeast (LMC 2) to the north (NGC 1818 at $\alpha=5^{\mathrm{h}} 04^{\mathrm{m}} 03^{\mathrm{s}}$, $\delta=-66^{\circ} 26^{\prime} 00^{\prime \prime}$ ) (de Boer et al. 1998). 30 Doradus has an age of 3-5 Myr, LMC 4 of 9-16 Myr, and NGC 1818 of 25-30 Myr (Parker \& Garmany 1993; Will et al. 1995; Braun et al. 1997; Grebel 1997). The difference in age between these shells corresponds to their distance along the border of the disk divided by the galaxy's rotation velocity (Mastropietro et al. 2009).

We find no evidence of such a cluster age distribution for the LMC. The older clusters are mostly located in the bar region and a few along the rim. Most clusters populating the LMC bar were adopted from the sample published by PU00. Ages of clusters older than $\sim 1$ Gyr cannot be derived because of the limited photometric depth of the MCPSs, which does not resolve the MSTOs of older clusters. Overall, our spatial cluster age distribution does not support ram pressure as the primary agent of cluster formation in the LMC.

As mentioned above, the highest concentration of young clusters can be found in the 30 Doradus region (NGC 2070) in the southeastern part of the LMC at roughly $\alpha=5^{\mathrm{h}} 38^{\mathrm{m}}, \delta=$ $-69^{\circ} 06^{\prime}$. In this large star-forming complex many SGSs are interlocking, where active star formation occurred simultaneously (Kim et al. 1999). A number of smaller shells have formed along the rims of the supershells, as expected for self-propagating star formation (e.g., McCray \& Kafatos 1987). The HII regions are sometimes associated with the HI shells, and the largest concentration can be found in the 30 Doradus region, as well as in SGSs hosting the youngest star clusters.

Figure 10 displays six snapshots showing the spatial distribution of LMC star clusters within different age bins. The first snapshot shows only clusters younger than $20 \mathrm{Myr}$. The star clusters are located in the 30 Doradus region, LMC 4, in the western part of the bar and along the rim in the southwestern part of the galaxy. Star clusters within the age range of $20 \mathrm{Myr}$ and $100 \mathrm{Myr}$ in the second and third snapshots are more widely distributed over the entire SGSs and in their intershell regions, as well as in the giant shells. There is no one-to-one correlation between the HI shells and the ionized gas traced out by the $\mathrm{H} \alpha$ regions (Mizuno et al. 2001a; Book et al. 2008). Along the bar, we now see pronounced activity in the western region and in the center, while the eastern part of the bar is essentially quiescent. Note also the particularly strong activity in the 30 Doradus region and in and around SGS 11 (LMC 4).

The LMC bar and the LMC rim are fully populated with star clusters within the age range of $100 \mathrm{Myr}$ and $250 \mathrm{Myr}$ (fourth snapshot). Star clusters with ages between $250 \mathrm{Myr}$ and 500 Myr mostly populate the LMC bar, but many are also distributed along the western and northwestern LMC rim, as well as in the northeast (fifth snapshot). Star clusters older than $500 \mathrm{Myr}$ (up to $1 \mathrm{Gyr}$ ) are mostly distributed along the LMC rim, while the bar region is essentially devoid of clusters in this age range. Also for the LMC, we find the older objects outside the shell regions and the younger objects are located along the rims and intercloud regions. The formation of young LMC clusters may have been triggered by shell expansion and interactions, which might be the reason we do not find evidence for the standard model of shell formation (McCray \& Kafatos 1987; see also Braun et al. 1997; Points et al. 1999). In the following we discuss the spatial distribution of the clusters of different ages inside the supershells SGS 3 (LMC 1), SGS 4 (LMC 8), SGS 9 (LMC 9), SGS 11 (LMC 4), SGS 7 (LMC 5), SGS 12 (LMC 3), and SGS 19 (LMC 2) in more detail.

SGS 3 (LMC 1) is located in the northwestern corner of the LMC at $\alpha=5^{\mathrm{h}} 00^{\mathrm{m}}, \delta=-65^{\circ} 40^{\prime}$ and has a dynamical age of $\sim 7 \mathrm{Myr}$ (Kim et al. 1999). Blue and red supergiants in the southern shell area were found to have ages below $30 \mathrm{Myr}$ (Grebel \& Brandner 1998). Young stellar objects are mostly distributed around the shell's southern periphery (Book et al. 2009) inside nearby HII regions. There is no cluster younger than $30 \mathrm{Myr}$ in our sample in SGS 3 (LMC 1), and only a few clusters within the age range of 30-100 Myr are located in the southeastern region. Clusters with ages between $30 \mathrm{Myr}$ and $500 \mathrm{Myr}$ are distributed over the entire projected area of shell. The clusters from our sample in this shell have a mean age of $\sim 150 \mathrm{Myr}$. Star formation in this shell mostly occurred in HII regions independent of the shell (Book et al. 2009). One OB association stretches from the center of the shell to a bright southeastern HII region, indicating a propagation of star formation. No indication of self-propagating star formation can be found in this study, which might be due to the incomplete sample. Given this age structure, the formation of the shell is unrelated to the comparatively much older star clusters.

$S G S 4$ (LMC 8) lies in the southwestern corner of the LMC at $\alpha=5^{\mathrm{h}} 03^{\mathrm{m}}, \delta=-70^{\circ} 30^{\prime}$ and has a dynamical age of $\sim 6 \mathrm{Myr}$ (Kim et al. 1999). This shell is clearly visible in the spatial distribution of supergiants (ages < 30 Myr, Grebel \& Brandner 1998). OB associations were found in the north and the east of the shell. SGS 4 (LMC 8) has a complex structure of HI lobes, which are connected (Book et al. 2008). Star clusters in this shell cover an age range between $\sim 12$ to $500 \mathrm{Myr}$ and have a mean age of 100 Myr. The northern, the northeastern, and the eastern lobes show expansion where a few clusters younger than $50 \mathrm{Myr}$ are also located (see Fig. 10). The southern lobes show no distinct expansion (Book et al. 2008), and in this region the shell appears to be essentially quiescent. A few clusters in the age range between $50 \mathrm{Myr}$ and $100 \mathrm{Myr}$ are found in the center and the northern region of the shell. Clusters in the age range betweeen $100 \mathrm{Myr}$ and $250 \mathrm{Myr}$ are located in the center, the east, and the northwest. The shell is mostly devoid of clusters older than 250 Myr. Only a few clusters are located along the rim regions in the east and the west. As for SGS 3 (LMC 1), no indication of self-propagating star formation is found in this study.

$S G S 9$ (LMC 9) is located in the southern part of the LMC at $\alpha=5^{\mathrm{h}} 25^{\mathrm{m}}, \delta=-70^{\circ} 05^{\prime}$. The shell is a collection of several giant 
K. Glatt et al.: Ages and luminosities of young SMC/LMC star clusters and the recent star formation history of the Clouds
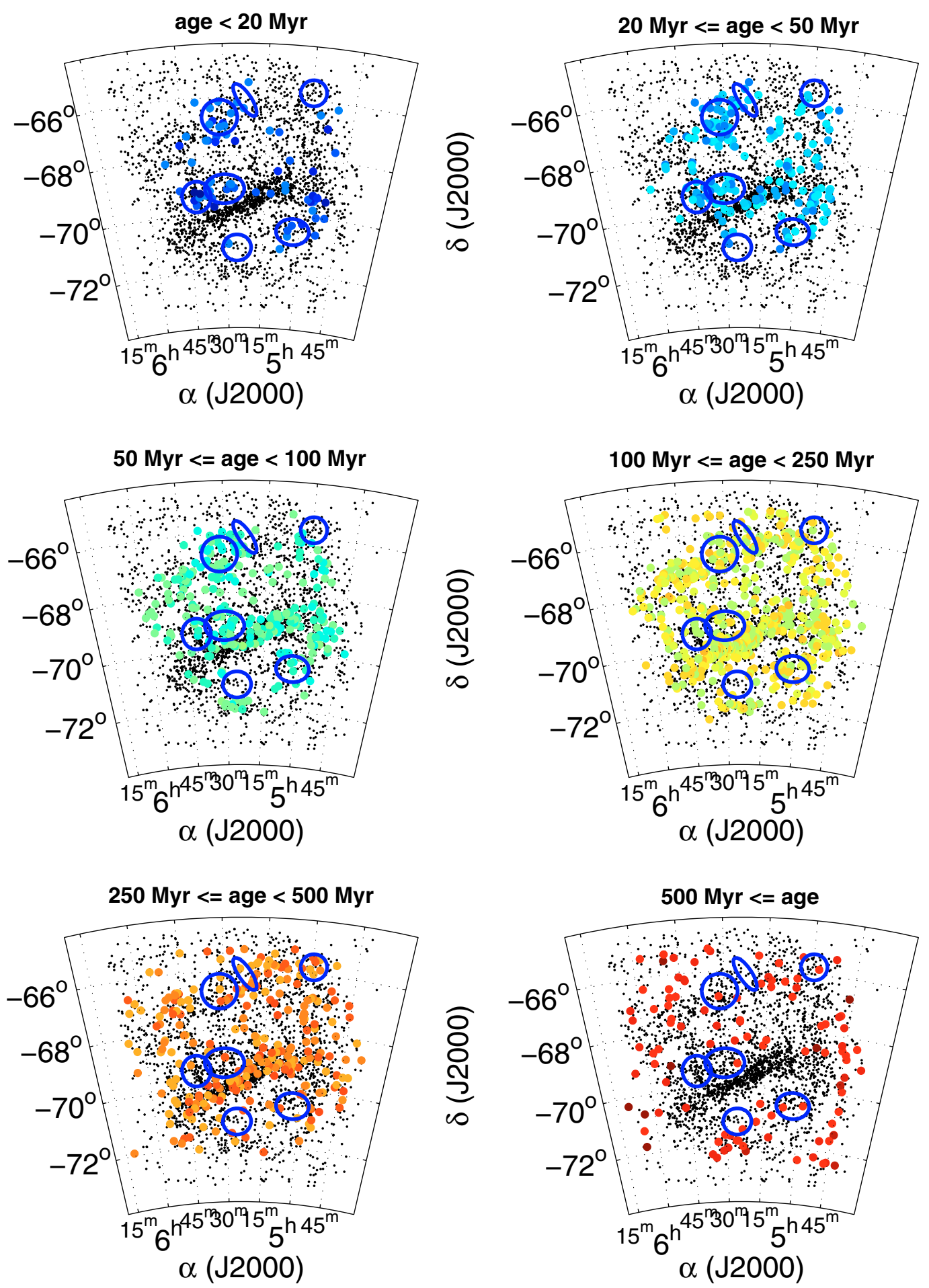

Fig. 10. Spatial distribution of LMC clusters younger than $1 \mathrm{Gyr}$ in different age bins.

$\mathrm{H} \alpha$ shells, but has no corresponding $\mathrm{HI}$ shell structure, which is why Book et al. (2008) characterized the shell as false. This shell has almost never shown cluster formation activity within the period studied here. HI supershells are distributed from west to northeast along the periphery. The shell showing the most cluster formation activity is a region in the southeast, outside the boundary of the shell. In this region many clusters with ages between $50 \mathrm{Myr}$ and $250 \mathrm{Myr}$ are found, which might be correlated with the expanding $\mathrm{H} \alpha$ and $\mathrm{HI}$ region. The few clusters younger than $50 \mathrm{Myr}$ are located at the northeastern shell rim, which might be associated with HII regions. This shell is probably very old, because almost no young clusters are located in this shell, and barely any supergiants and Cepheids were found in the shell region (Grebel \& Brandner 1998). The star clusters from our sample in this shell have ages between 20 Myr to 1 Gyr with a mean age of $170 \mathrm{Myr}$.

SGS 11 (LMC 4) is the largest SGS in the LMC and is located at $\alpha=5^{\mathrm{h}} 32^{\mathrm{m}}, \delta=-66^{\circ} 40^{\prime}$. The shell is in collision with SGS 7 (LMC 5). The densest part of the shell lies at the northwestern border in the region between SGS 11 (LMC 4) and SGS 7 (LMC 5). Multiple OB associations exist in HII regions in the interaction zone, along the shell rim, and near the center 
of the shell (Book et al. 2009). Yamaguchi et al. (2001a) find the clusters at the rim of SGS 11 (LMC 4) to be associated with CO clouds. They are mostly noticeable in the interaction region. Stars inside the shell were found to be $\sim 9-16 \mathrm{Myr}$ old, while those at the rim are $\$ 6 \mathrm{Myr}$ old (Braun et al. 1997). The analysis of Cepheids and other supergiants shows that star formation in this shell started about $25 \mathrm{Myr}$ ago in its center region and then slowly moved out toward the outer rims (Grebel \& Brandner 1998). SGS 11 (LMC 4) appears to be the only shell that shows a weak indication of propagating star formation and has formed only within the past $10 \mathrm{Myr}$. Star clusters from our sample cover an age range between 12 and 500 Myr. The mean age lies at $60 \mathrm{Myr}$. Star clusters with ages less than $20 \mathrm{Myr}$ are found along the shell rim where the HII regions were also identified (Book et al. 2009). Star clusters with ages between 50-250 Myr are widely distributed across the entire shell. Clusters older than $250 \mathrm{Myr}$ are mostly found at the southern rim of the shell. Two are found in the center region and a single one at the northern rim. If the very young age of this shell is correct, the formation of clusters older than $\sim 20 \mathrm{Myr}$ is not related to SGS 11 (LMC 4). No indication of self-propagating star formation is found in this study.

SGS 7 (LMC 5) is located to the northwest of SGS 11 (LMC 4) at $\alpha=5^{\mathrm{h}} 22^{\mathrm{m}}, \delta=-66^{\circ} 00^{\prime}$ and has a dynamical age of $\sim 5 \mathrm{Myr}$ (Kim et al. 1999). The shell is in collision with SGS 11 (LMC 4), which caused a density enhancement on the eastern side of the shell. The cluster age distribution is similar to the one in SGS 11 (LMC 4). The star clusters from our sample in this shell have ages between 20 to $400 \mathrm{Myr}$ with a mean age of 130 Myr. Only five clusters from our sample younger than $100 \mathrm{Myr}$ are found in this shell, and three of them are located at the eastern rim. No OB associations are found inside the shell, but along the interaction zone and along the rim (Book et al. 2009). No OB association was detected in the shell interior. The shell has a relatively high CO concentration (Yamaguchi et al. 2001b). The highest CO density is located in the interaction zone, superposed on $\mathrm{OB}$ associations and corresponding $\mathrm{HII}$ regions. Two more high-density $\mathrm{CO}$ regions are located in the southern region of the interaction zone and in the north. Only very few objects younger than $20 \mathrm{Myr}$ were found in the present sample because of our object selection. The southern part of the shell and the surroundings are covered by star clusters with ages between 100-500 Myr. The shell is devoid of objects older than 500 Myr. Like SGS 11 (LMC 4), this shell is probably very young. Especially in the interaction zone, where SGS 7 (LMC 5) and SGS 11 (LMC 4) are colliding, a large number of blue and red supergiants are located, indicating recent star formation (Grebel \& Brandner 1998). The old clusters in the south of the shell might have formed in a shell that is dissolved already or via a nonshell-related mechanism. Star formation in this shell is probably triggered by shell expansion, especially in the collision zone.

SGS 12 (LMC 3) is located at $\alpha=5^{\mathrm{h}} 30^{\mathrm{m}}, \delta=-69^{\circ} 00^{\prime}$ and is in collision with SGS 19 (LMC 2). The large star-forming region 30 Doradus lies in the interaction zone. The SGS is divided into northern and southern lobes and shows significant $\mathrm{HI}$ and $\mathrm{H} \alpha$ structures (Book et al. 2008). Old OB associations (age $\gtrsim 12$ Myr) exist in the interior of the shell, while young OB associations (age $\lesssim 12 \mathrm{Myr}$ ) are found within dense HII regions. The cluster mean age of this shell lies at $\sim 75 \mathrm{Myr}$, and they cover an age range of $5 \mathrm{Myr}$ to 1 Gyr. Clusters younger than $20 \mathrm{Myr}$ are concentrated in the center region of the shell, and those with ages between 20-250 Myr are more widely distributed. The shell barely contains any clusters older than 250 Myr. No signs of propagating star formation events are found. Clusters might have formed via shell expansion, most efficiently in the collision zone. Looking at the age distribution of blue and red supergiants (age $<30 \mathrm{Myr}$ ), the intershell region is clearly visible as a region with a high concentration of these objects (Grebel \& Brandner 1998). The oldest supergiants may have triggered the subsequent compression of dense molecular clouds and resulting star bursts.

SGS 19 (LMC 2) is located to the southeast of 30 Doradus at $\alpha=5^{\mathrm{h}} 44^{\mathrm{m}}, \delta=-69^{\circ} 20^{\prime}$ and is the brightest SGS in the LMC in $\mathrm{H} \alpha$ (Kim et al. 1999). The shell is colliding with SGS 12 (LMC 3). It has very complex structures in $\mathrm{HI}$ and $\mathrm{H} \alpha$ and shows star-forming regions on its western edge (Book et al. 2008). The clusters from our sample cover an age range between $40 \mathrm{Myr}$ and 1 Gyr. The cluster mean age lies at $\sim 250$ Myr. Clusters younger than $50 \mathrm{Myr}$ are located in the center of the shell and toward 30 Doradus. The shell only hosts a few clusters with ages between $50 \mathrm{Myr}$ and $250 \mathrm{Myr}$ and is almost devoid of older clusters. Twenty-nine clusters in the direction of LMC 2 were reported for which ages have been determined (e.g., Elson \& Fall 1985; Bica et al. 1996). Eleven of them are younger than $10 \mathrm{Myr}$, seven have ages between 10-30 Myr, one has an age of 30-70 Myr, and one is significantly older with an age of 400-800 Myr. The youngest clusters are associated with HII regions that are located along the periphery of LMC 2 (Points et al. 1999). The analysis of supergiants reveals that this shell is very young (10-15 Myr, Grebel \& Brandner 1998), and in the spatial distribution of supergiants the interaction region between SGS 19 (LMC 2) and SGS 12 (LMC 3) is clearly visible.

Mizuno et al. (2001b) find that most of the CO clouds in the LMC are distributed in dense parts of HI gas and over several HII regions. LMC CO clouds are often located at the outer edge of SGSs as for, e.g., SGS 11 (LMC 4) (Yamaguchi et al. 2001b). Furthermore these authors find that clusters associated with the CO clouds are younger than $5 \mathrm{Myr}$, which we cannot investigate owing to the limited age range of the sample.

\subsection{Dissolution effects}

Because of the different morphology of the MCs compared to the MW and the absence of differential rotation of SMC and LMC, low-concentration clusters can easily survive in these two galaxies, while the MW rotates differentially, which leads to shear and interaction with massive molecular clouds that can result in cluster dissolution. For the LMC, Parmentier \& de Grijs (2008) find a cluster formation rate of 0.3 clusters $\mathrm{Myr}^{-1} 5 \mathrm{Gyr}$ ago, which increased steadily to a present rate of $\sim 25$ clusters $\mathrm{Myr}^{-1}$. Such studies have not been published for the SMC yet. The main distinct phases and corresponding typical timescales of cluster disruption in the MCs are: (I.) infant mortality $\left(\sim 10^{7} \mathrm{yr}\right)$; (II.) mass loss dominated by stellar evolution $\left(\sim 10^{8} \mathrm{yr}\right)$; and (III.) a phase dominated by tidal relaxation $\left(\sim 10^{9} \mathrm{yr}\right)$ in which the mass loss is driven by the clusters' dynamical evolution and external influence of the tidal field of the host galaxy (Lamers et al. 2005; Bastian \& Gieles 2008, and references therein). Additionally, tidal external perturbations speed up the process of disruption during all three phases, but these effects operate on longer timescales and mostly affect phase III. Most of the clusters in our sample have survived the first phase and are dominated by the second and third phases. The rate of infant mortality is highly dependent on the ambience of the host galaxy, but is largely mass-independent - at least for masses in excess of $\sim 10^{4} M_{\odot}$ (e.g., Bastian et al. 2005). Infant mortality is mainly driven by gas expulsion. This lifecycle of a cluster results from the expulsion of the intracluster gas owing to explosive expansion 
K. Glatt et al.: Ages and luminosities of young SMC/LMC star clusters and the recent star formation history of the Clouds
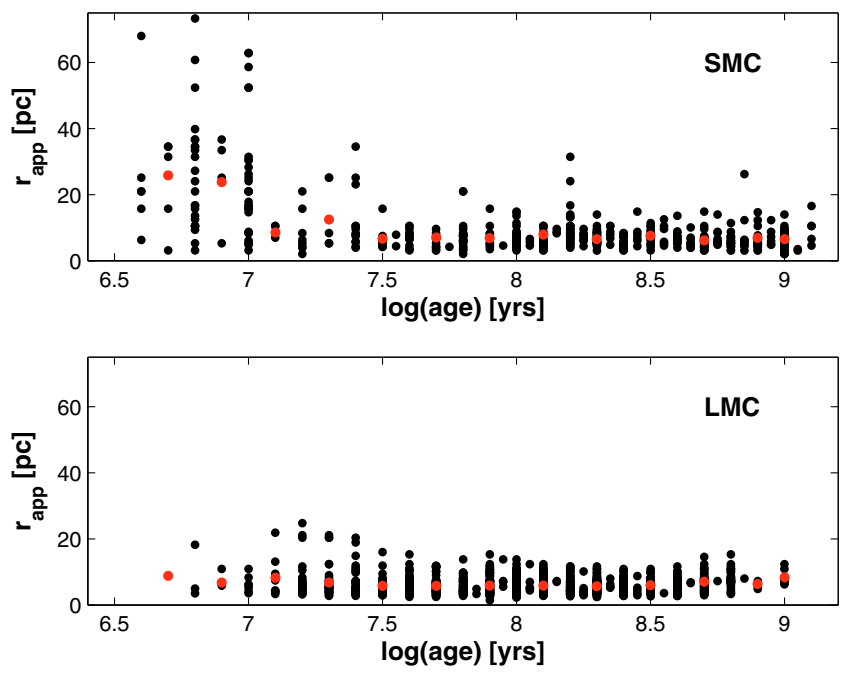

Fig. 11. Age vs. apparent radius. In both SMC and LMC, no indication of cluster dissolution is visible. The large radii for associations in the $r$ vs. age distribution of the SMC come from C06. The red dots represent the mean of the radius distribution.

driven by stellar winds or supernova activity (e.g., Mengel et al. 2005; Bastian \& Goodwin 2006; Goodwin \& Bastian 2006). The end of cluster infant mortality depends on the crossing time of the cluster's gaseous progenitor and on the external tidal field (Parmentier 2009). The denser the gaseous precursor of the star cluster, the shorter the cluster's crossing time and the quicker the cluster's violent relaxation. The stronger the external tidal field, the smaller the cluster's tidal radius and the faster unbound stars leave the cluster. Because the external tidal fields of the MCs are not very strong, most star clusters in the MCs surviving the phase of infant mortality will have returned to an equilibrium state after 40-50 Myr (Goodwin \& Bastian 2006). Therefore, some of the clusters in the present sample might dissolve and not survive the first phase of cluster dissruption, while most clusters have ages of $40 \mathrm{Myr}$ and higher, placing them in the phase where stellar evolution has become a major agent of change, whereas the gas has already been expelled.

The second (mass-dependent) lifecycle of a cluster includes the so-called secular evolution (gas-free evolution). This phase is driven by 2-body relaxation and the morphology of the host galaxy. Therefore, the disruption time of a cluster is dependent on the internal cluster conditions, such as the initial mass, density, and velocity dispersion, as well as on external conditions, such as the orbit in the galaxy and tidal heating (e.g., Boutloukos \& Lamers 2003; Lamers et al. 2005; Parmentier \& de Grijs 2008).

Figure 11 shows the age-radius relation of the combined SMC and LMC cluster sample with ages between 5 Myr and 1 Gyr. The SMC and LMC cluster ages are plotted against their apparent overall radii (Eq. (1)) from the combined samples derived in this study and in C06 (SMC) and PU00 (LMC). The youngest objects in the SMC have radii up to $\sim 4$ arcmin, but these objects are clusters adopted from C06 and are mostly classified as associations. Moreover, until an age of 40 Myr, star clusters in the MCs can still undergo infant mortality (Goodwin \& Bastian 2006), so we have to treat this subsample with caution. The radii decrease until an age of $\sim 25 \mathrm{Myr}$ and then remain relatively constant until 1 Gyr. Because the youngest objects in the LMC were not age-dated, no extended objects can be seen at the young age end of the distribution. The cluster radii in the LMC
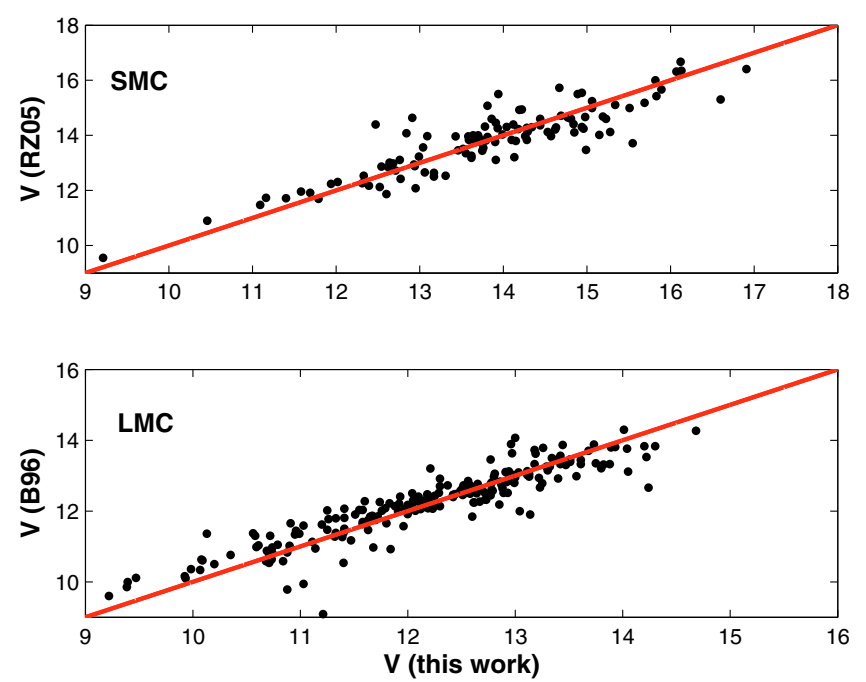

Fig. 12. $V$ band magnitudes derived in this work compared with the $V$ band magnitudes derived by RZ05 (SMC) and by B96 (LMC) for the clusters in common. The solid line is unity.

show the same behavior as the SMC clusters and are constant with time during the analyzed period. The cluster disruption time for the SMC and the LMC derived in the literature is on the order of $8 \times 10^{9}$ Myr (e.g., Boutloukos \& Lamers 2003; Parmentier \& de Grijs 2008).

We have shown (Glatt et al. 2009) that clusters with ages older than 1 Gyr have a trend to larger core radii with increasing age. This trend was first found by Mackey \& Gilmore (2003a) and is also visible for LMC, Fornax, and Sagittarius clusters (Mackey \& Gilmore 2003b,c). We note, however, that the radii in Fig. 11 are apparent overall radii (see Sect. 4) so that we cannot draw any firm conclusions on possible dissolution effects based on the observed sizes alone.

\section{The cluster luminosities}

RZ05 published luminosities of 204 SMC star clusters measuring integrated colors from the MCPS. B96 published integrated photometry of 624 LMC star clusters that was based on observations carried out at the CTIO in Chile and at the CASLEO in Argentina. For the age-dated clusters in our samples, we computed luminosities by summing the flux of each star within the individual cluster radii (Eq. (1)). To correct for field stars, the luminosity of a concentric annulus between 2 and 1 arcmin was calculated for each cluster, area-corrected, and subtracted from the cluster luminosity. The 112 clusters in our sample are in common with RZ05's sample and 217 clusters with B96's sample. The comparison between the $V$ band luminosity derived in our study with RZ05 and B96 (Fig. 12) shows that the $V$ band magnitudes are overall in good agreement. The LMC $V$ band magnitudes $<12$ mag published by B96 are systematically fainter by a small amount than the ones computed in this study. On the other hand, magnitudes $(V>13.50 \mathrm{mag})$ by B96 appear to be slightly brighter at the faint end the $V$ band. The dispersion about the $1: 1$ relation for all clusters in common is $\sigma_{V}=0.28$ (SMC) and 0.22 (LMC).

The younger the star clusters, the brighter they are because young clusters still contain very massive hot stars (e.g., supergiants), which contribute most of the light. The older a cluster gets, the more of these massive stars are in their end stage of evolution so can no longer contribute to the cluster light. Therefore, 

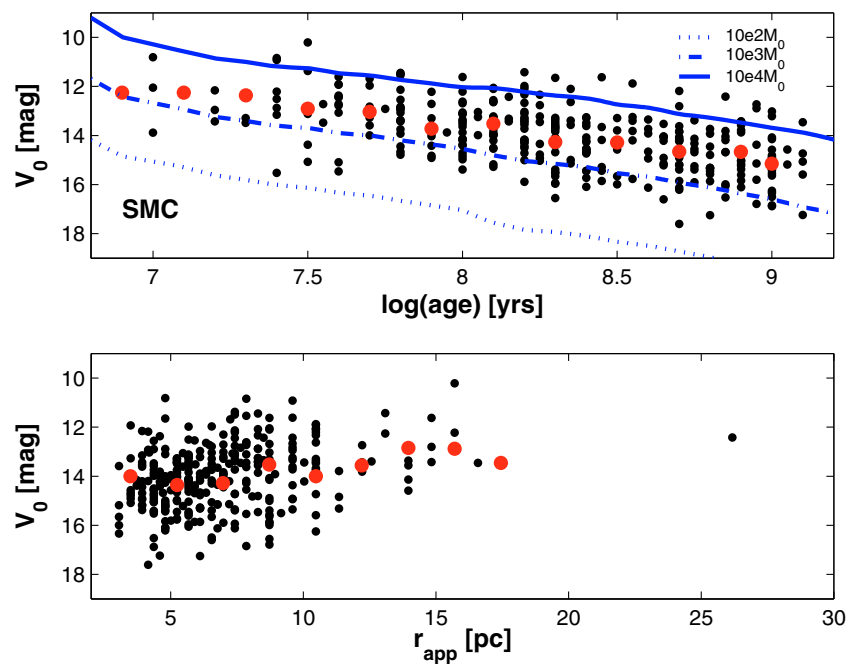

Fig. 13. $V_{0}$ vs. $\log$ (age) and $V_{0}$ vs. apparent overall radius for our SMC clusters. The luminosities were derived in this study. The red dots represent the mean of the age distribution in magnitude bins of 0.3 mag. The trend to fainter magnitudes with increasing age is obvious. The blue lines represent three GALEV models (Kotulla et al. 2009) of different total mass: $10^{4} M_{\odot}$ (solid), $10^{3} M_{\odot}$ (dash-dotted), and $10^{2} M_{\odot}$ (dotted).
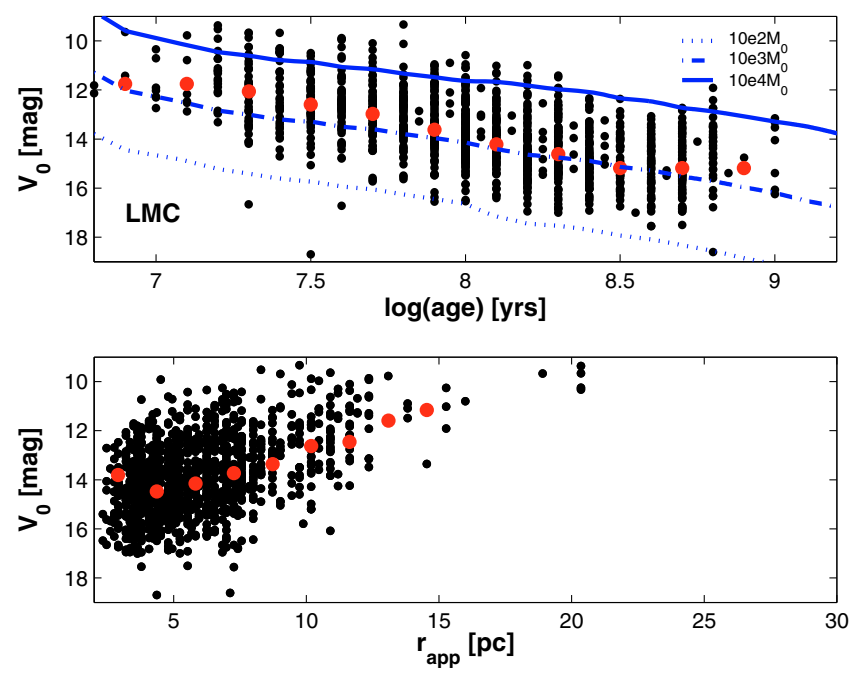

Fig. 14. $V_{0}$ vs. $\log ($ age $)$ and $V_{0}$ vs. apparent overall radius relations for our LMC clusters. The luminosities were derived in this study. The red dots represent the mean of the age distribution in magnitude bins of $0.3 \mathrm{mag}$. The trend to fainter magnitudes with increasing age is also obvious in this plot. The blue lines represent three GALEV models (Kotulla et al. 2009) of different total mass: $10^{4} M_{\odot}$ (solid), $10^{3} M_{\odot}$ (dash-dotted), and $10^{2} M_{\odot}$ (dotted).

clusters become fainter and redder with increasing age. This effect can be seen in the upper panels of Figs. 13 (SMC) and 14 (LMC), in which cluster age is plotted versus luminosity. The mean of the age distribution in magnitude bins is of 0.3 mag. We overplotted three GALEV models (Kotulla et al. 2009) for the total cluster masses of $10^{2} M_{\odot}, 10^{3} M_{\odot}$, and $10^{4} M_{\odot}$ using a Salpeter initial mass function. GALEV is a model for computing the spectral evolution of single stellar populations and galaxies. The GALEV models give an overview of the cluster mass range. The lower plots of Figs. 13 and 14 show that star clusters of the SMC and LMC become brighter with increasing radius, which is expected assuming a larger number of stars within the cluster radius contributing to the total cluster luminosity.

\section{Summary}

We have presented ages and luminosities of 324 and 1193 populous SMC and LMC star clusters, respectively. An age range of $\sim 9$ Myr to 1 Gyr was covered based on isochrone fitting to resolved color-magnitude diagrams in both galaxies. Using only the cluster ages derived in this study, we find two maxima of enhanced cluster formation for both galaxies, which appear to be correlated. In the SMC, the peaks are found at $\sim 160 \mathrm{Myr}$ and $\sim 630 \mathrm{Myr}$, and in the LMC at $\sim 125 \mathrm{Myr}$ and $\sim 800 \mathrm{Myr}$. Model calculations predict that the last close encounter between LMC and SMC occurred around 100-200 Myr ago. During a close encounter, the star formation is expected to be enhanced. Therefore, the first peaks in the cluster age distributions could have been triggered by this tidal interaction. Extending our samples with cluster ages derived by $\mathrm{C} 06$, we find a third pronounced period of enhanced cluster formation in the SMC at around 8 Myr. We find the same in the LMC by combining our sample with the one of PU00. These peaks are only visible if we extend our sample with objects classified as associations, objects that did not or could not reach higher ages because they dissolved too quickly.

The youngest objects in both galaxies are associated with super giant shells, giant shells, the intershell region, and with HII regions. Their formation is probably related to shell expansion and shell interaction. In the spatial distribution of the clusters younger than $\sim 16$ Myr the two SMC shells are clearly visible. The older objects are widely spread across the entire SMC main body, but show a concentration in the western part of the galaxy. In the LMC, the youngest objects are concentrated in 30 Doradus, SGS 11 (LMC 4), and in the giant shells located in the western part and in the bar region. The older LMC clusters are mostly distributed along the bar and along the rim. One can see nicely how star cluster formation propagated along the LMC bar. We find no indication of propagating star cluster formation in the SGSs in either LMC or SMC. Most of the LMC star clusters are older than the dynamical ages of the SGSs and therefore may have formed in shells that have already dissolved and cannot be detected at the present day.

No obvious dissolution effects were found for MCs star clusters younger than $\sim 1$ Gyr. It is quite difficult to ascertain any real absence of cluster dissolution using this study. Two biases may play a major role. 1 . Infant mortality cannot be accounted for, because very young star clusters and OB-associations are not included in our sample, and 2 . cluster dissolution processes for clusters are older than $\sim 1$ Gyr, because we did not age-date clusters in this age range. Within the time period considered here - 10 Myr to $1 \mathrm{Gyr}$ - we do not find any evidence of cluster dissolution.

In both galaxies, the clusters become fainter with increasing age. The very massive hot stars, which are still present in the young star clusters and contribute most of the light, become fainter and redder with increasing age, as do the star clusters. This trend can be seen in both the LMC and the SMC. The total cluster luminosity increases with increasing radius due to a larger number of stars within the cluster radius.

Acknowledgements. We thank the anonymous referee for extremely useful suggestions for improving our paper. We gratefully acknowledge support by the Swiss National Science Foundation through grant number 200020-105260 and 200020-113697. Andreas Koch acknowledges support by an STFC postdoctoral fellowship. 
K. Glatt et al.: Ages and luminosities of young SMC/LMC star clusters and the recent star formation history of the Clouds

\section{References}

Alves, D. R. 2004, New Astron. Rev., 48, 659

Bastian, N., \& Gieles, M. 2008, in Mass Loss from Stars and the Evolution of Stellar Clusters, ed. A. de Koter, L. J. Smith, \& L. B. F. M. Waters, ASP Conf Ser., 388, 353

Bastian, N., \& Goodwin, S. P. 2006, MNRAS, 369, L9

Bastian, N., Gieles, M., Lamers, H. J. G. L. M., Scheepmaker, R. A., \& de Grijs, R. 2005, A\&A, 431, 905

Baumgardt, H., \& Makino, J. 2003, MNRAS, 340, 227

Bekki, K., \& Chiba, M. 2005, MNRAS, 356, 680

Bekki, K., Couch, W. J., Beasley, M. A., et al. 2004, ApJ, 610, L93

Bertelli, G., Mateo, M., Chiosi, C., \& Bressan, A. 1992, ApJ, 388, 400

Bertelli, G., Bressan, A., Chiosi, C., Fagotto, F., \& Nasi, E. 1994, A\&AS, 106, 275

Bica, E., \& Dutra, C. M. 2000, AJ, 119, 1214

Bica, E. L. D., \& Schmitt, H. R. 1995, ApJS, 101, 41

Bica, E., Claria, J. J., Dottori, H., Santos, Jr., J. F. C., \& Piatti, A. E. 1996, ApJS, 102,57

Bica, E., Bonatto, C., Dutra, C. M., \& Santos, J. F. C. 2008, MNRAS, 389, 678

Book, L. G., Chu, Y., \& Gruendl, R. A. 2008, ApJS, 175, 165

Book, L. G., Chu, Y., Gruendl, R. A., \& Fukui, Y. 2009, AJ, 137, 3599

Boutloukos, S. G., \& Lamers, H. J. G. L. M. 2003, MNRAS, 338, 717

Braun, J. M., Bomans, D. J., Will, J., \& de Boer, K. S. 1997, A\&A, 328, 167

Bruhweiler, F. C., Gull, T. R., Kafatos, M., \& Sofia, S. 1980, ApJ, 238, L27

Chiosi, E., Vallenari, A., Held, E. V., Rizzi, L., \& Moretti, A. 2006, A\&A, 452, 179

Crowl, H. H., Sarajedini, A., Piatti, A. E., et al. 2001, AJ, 122, 220

Davies, R. D., Elliott, K. H., \& Meaburn, J. 1976, MmRAS, 81, 89

de Boer, K. S., Braun, J. M., Vallenari, A., \& Mebold, U. 1998, A\&A, 329, L49

de Grijs, R., \& Goodwin, S. P. 2009, in IAU Symp. 256, ed. J. T. van Loon, \&

J. M. Oliveira, 311

Dieball, A., Müller, H., \& Grebel, E. K. 2002, A\&A, 391, 547

Dotter, A., Chaboyer, B., Jevremović, D., et al. 2007, AJ, 134, 376

Elmegreen, B. G., \& Chiang, W. 1982, ApJ, 253, 666

Elmegreen, B. G., \& Efremov, Y. N. 1997, ApJ, 480, 235

Elson, R. A. W., \& Fall, S. M. 1985, ApJ, 299, 211

Gardiner, L. T., \& Noguchi, M. 1996, MNRAS, 278, 191

Girardi, L., Chiosi, C., Bertelli, G., \& Bressan, A. 1995, A\&A, 298, 87

Girardi, L., Bertelli, G., Bressan, A., et al. 2002, A\&A, 391, 195

Glatt, K., Gallagher, III, J. S., Grebel, E. K., et al. 2008a, AJ, 135, 1106

Glatt, K., Grebel, E. K., Sabbi, E., et al. 2008b, AJ, 136, 1703

Glatt, K., Grebel, E. K., Gallagher, J. S., et al. 2009, AJ, 138, 1403

Gonzalez, G., \& Wallerstein, G. 1999, AJ, 117, 2286

Goodwin, S. P., \& Bastian, N. 2006, MNRAS, 373, 752

Grebel, E. K. 1997, A\&A, 317, 448

Grebel, E. K., \& Brandner, W. 1998, in The Magellanic Clouds and Other Dwarf Galaxies, ed. T. Richtler, \& J. M. Braun (Aachen: Shaker), 151

Grebel, E. K., \& Richtler, T. 1992, A\&A, 253, 359

Grebel, E. K., Roberts, W. J., \& Brandner, W. 1996, A\&A, 311, 470

Grebel, E. K., Zaritsky, D., Harris, J., \& Thompson, I. 1999, in New Views of the Magellanic Clouds, ed. Y.-H. Chu, N. Suntzeff, J. Hesser, \& D. Bohlender, IAU Symp., 190, 405

Harris, J., \& Zaritsky, D. 2001, ApJS, 136, 25

Hatzidimitriou, D., Cannon, R. D., \& Hawkins, M. R. S. 1993, MNRAS, 261, 873

Hill, V. 1999, A\&A, 345, 430

Hill, V., Barbuy, B., \& Spite, M. 1997, A\&A, 323, 461

Holtzman, J. A., Gallagher, III, J. S., Cole, A. A., et al. 1999, AJ, 118, 2262

Johnson, J. A., Bolte, M., Stetson, P. B., Hesser, J. E., \& Somerville, R. S. 1999, ApJ, 527, 199

Kallivayalil, N., van der Marel, R. P., \& Alcock, C. 2006a, ApJ, 652, 1213

Kallivayalil, N., van der Marel, R. P., Alcock, C., et al. 2006b, ApJ, 638, 772

Keller, S. C., \& Wood, P. R. 2006, ApJ, 642, 834
Kim, S., Dopita, M. A., Staveley-Smith, L., \& Bessell, M. S. 1999, AJ, 118, 2797

Kim, S., Staveley-Smith, L., Dopita, M. A., et al. 2003, ApJS, 148, 473

Kotulla, R., Fritze, U., Weilbacher, P., \& Anders, P. 2009, MNRAS, 396, 462

Lah, P., Kiss, L. L., \& Bedding, T. R. 2005, MNRAS, 359, L42

Lamers, H. J. G. L. M., Gieles, M., \& Portegies Zwart, S. F. 2005, A\&A, 429, 173

Lançon, A., \& Mouhcine, M. 2000, in Massive Stellar Clusters, ed. A. Lançon, \& C. M. Boily, ASP Conf. Ser., 211, 34

Larson, R. B. 1993, in The Globular Cluster-Galaxy Connection, ed. G. H. Smith, \& J. P. Brodie, ASP Conf. Ser., 48, 675

Lejeune, T., \& Schaerer, D. 2001, A\&A, 366, 538

Luck, R. E., Moffett, T. J., Barnes, III, T. G., \& Gieren, W. P. 1998, AJ, 115, 605

Mackey, A. D., \& Gilmore, G. F. 2003a, MNRAS, 338, 120

Mackey, A. D., \& Gilmore, G. F. 2003b, MNRAS, 338, 85

Mackey, A. D., \& Gilmore, G. F. 2003c, MNRAS, 340, 175

Mastropietro, C., Burkert, A., \& Moore, B. 2009, MNRAS, 399, 2004

Mathewson, D. S., Ford, V. L., \& Visvanathan, N. 1988, ApJ, 333, 617

McCray, R., \& Kafatos, M. 1987, ApJ, 317, 190

McKee, C. F., \& Ostriker, E. C. 2007, ARA\&A, 45, 565

Meaburn, J. 1980, MNRAS, 192, 365

Mengel, S., Lehnert, M. D., Thatte, N., \& Genzel, R. 2005, A\&A, 443, 41

Mighell, K. J., Sarajedini, A., \& French, R. S. 1998, AJ, 116, 2395

Mizuno, N., Rubio, M., Mizuno, A., et al. 2001a, PASJ, 53, L45

Mizuno, N., Yamaguchi, R., Mizuno, A., et al. 2001b, PASJ, 53, 971

Olsen, K. A. G., Hodge, P. W., Mateo, M., et al. 1998, MNRAS, 300, 665

Pagel, B. E. J., \& Tautvaišienè, G. 1999, Ap\&SS, 265, 461

Parisi, M. C., Grocholski, A. J., Geisler, D., Sarajedini, A., \& Clariá, J. J. 2009, AJ, 138, 517

Parker, J. W., \& Garmany, C. D. 1993, AJ, 106, 1471

Parmentier, G. 2009 [arXiv: 0901.3140]

Parmentier, G., \& de Grijs, R. 2008, MNRAS, 383, 1103

Piatti, A. E., Santos, J. F. C., Clariá, J. J., et al. 2001, MNRAS, 325, 792

Pietrzynski, G., \& Udalski, A. 1999, Acta Astron., 49, 157

Pietrzynski, G., \& Udalski, A. 2000, Acta Astron., 50, 355

Pietrzynski, G., Udalski, A., Kubiak, M., et al. 1998, Acta Astron., 48, 175

Points, S. D., Chu, Y. H., Kim, S., et al. 1999, ApJ, 518, 298

Rafelski, M., \& Zaritsky, D. 2005, AJ, 129, 2701

Rich, R. M., Shara, M., Fall, S. M., \& Zurek, D. 2000, AJ, 119, 197

Romaniello, M., Primas, F., Mottini, M., et al. 2005, A\&A, 429, L37

Stanimirovic, S., Staveley-Smith, L., Dickey, J. M., Sault, R. J., \& Snowden, S. L. 1999, MNRAS, 302, 417

Staveley-Smith, L., Sault, R. J., Hatzidimitriou, D., Kesteven, M. J., \& McConnell, D. 1997, MNRAS, 289, 225

Storm, J., Carney, B. W., Gieren, W. P., et al. 2004, A\&A, 415, 531

Subramaniam, A., \& Subramanian, S. 2009, ApJ, 703, L37

Tomisaka, K., Habe, A., \& Ikeuchi, S. 1981, Ap\&SS, 78, 273

Udalski, A. 1998, Acta Astron., 48, 383

Udalski, A., Szymanski, M., Kubiak, M., et al. 1998, Acta Astron., 48, 147

Udalski, A., Soszynski, I., Szymanski, M., et al. 1999, Acta Astron., 49, 223

Venn, K. A. 1999, ApJ, 518, 405

Whitmore, B. C. 1999, in Galaxy Interactions at Low and High Redshift, ed. J. E. Barnes, \& D. B. Sanders (Dordrecht: Kluwer), IAU Symp., 186, 251

Will, J., Bomans, D. J., \& de Boer, K. S. 1995, A\&A, 295, 54

Yamaguchi, R., Mizuno, N., Onishi, T., Mizuno, A., \& Fukui, Y. 2001a, ApJ, 553, L185

Yamaguchi, R., Mizuno, N., Onishi, T., Mizuno, A., \& Fukui, Y. 2001b, PASJ, 53, 959

Yoshizawa, A. M., \& Noguchi, M. 2003, MNRAS, 339, 1135

Zaritsky, D., Harris, J., \& Thompson, I. 1997, AJ, 114, 1002

Zaritsky, D., Harris, J., Thompson, I. B., Grebel, E. K., \& Massey, P. 2002, AJ, 123,855

Zaritsky, D., Harris, J., Thompson, I. B., \& Grebel, E. K. 2004, AJ, 128, 1606

Zhang, Q., Fall, S. M., \& Whitmore, B. C. 2001, ApJ, 561, 727

Zhao, H., \& Evans, N. W. 2000, ApJ, 545, L35 